This paper is a preprint of a paper submitted to and accepted for publication in IET Communications and is subject to Institution of Engineering and Technology Copyright.

The copy of record is available at IET Digital Library.

Some final publisher edits and corrections may not be included in this file.

\title{
Twenty Years of Ultrawideband: Opportunities and Challenges
}

Vit Sipal ${ }^{1}$, Ben Allen ${ }^{2}$, David Edwards ${ }^{1}$, Bahram Honary ${ }^{3}$

${ }^{1}$ Department of Engineering Science, Parks Road, University of Oxford, OX1 3PJ Oxford, UK, vit.sipal@eng.ox.ac.uk, david.edwards@eng.ox.ac.uk

${ }^{2}$ Centre for Wireless Research, University of Bedfordshire, Park Square, Luton LU1 3JU, ben.allen@beds.ac.uk

${ }^{3}$ Department of Communication Systems, Lancaster University, Lancaster, UK b.honary@lancaster.ac.uk

\section{Abstract}

It has been 20 years since the word ultrawideband has first been used in open literature. In these 20 years, ideas have been transformed into real products. Yet some might object that ultrawideband has not yet lived up to the promises of gigabit wireless. This review shows that despite some drawbacks, ultrawideband is not only needed because of the spectrum gridlock but it still can deliver its promises including gigabit wireless. To do so the gap between the potential, which is achievable, and the current performance must be closed. Thus, this review identifies some main issues of UWB (range, BER performance, data-rate, chip complexity and issues associated by strong narrowband interference). It shows that their reasons are well understood and addressed by erudite research which includes low complexity chip design, alternative modulation schemes, multiple antenna systems, UWB radio-over-fibre, higher band UWB and interferer detection and suppression techniques. A specific feature of this review is the cross-layer approach. The latest findings from different system layers, e.g., wave propagation, are linked to the system performance or complexity.

\section{Aims and structure}

The ultrawideband wireless communication technology for civilian use has been subject to intensive scientific investigation for the last two decades. At the time of writing this article, the search for "ultrawideband" and "UWB" in IEEExplore ${ }^{\mathrm{TM}}$ gives 860 journal papers and 1560 conference papers between 1991 and 2011. This amount of scientific work has resulted in serious progress from theoretical considerations to real products in the market place. However, the intensity of research activities is not being reduced as there is still a gap between the theoretical potential and the actual performance of practical systems. The main objective of this paper is to show that this gap is well understood and how on-going research activities aim to close it. 
As the gap must be identified first, the paper begins with explanation of the need for ultrawideband wireless communication (Section 2.1) followed by brief summary of the history of the technology (Section 2.2) and its current situation, achievements and issues which are thought to be the reason for the existence of the gap (Section 2.3). Section 3 then introduces the current UWB regulations in the leading markets to provide more understanding for the framework of UWB systems and also to introduce some recent changes which for instance increased the need for development of efficient detect and avoid algorithms. Section 4 then identifies range, BER performance, data-rate, chip complexity and issues associated by strong narrowband interference as the most burning issues of current UWB. Section 5 then groups current research activities in six subsets, describes them and shows how these activities aim to address one or more issues listed in section 4.

\section{Introduction to UWB}

\subsection{The need and the concept}

The motivation for ultrawideband wireless communication has been the inevitable destiny of wireless communication. In 1888 Heinrich Hertz demonstrated the existence of electromagnetic waves and so he supported the theoretical work of James Clerk Maxwell [1]. Despite the fact that Hertz "saw no practical use in his discovery", already in 1895 Guglielmo Marconi demonstrated wireless telegraphy over a distance of $1.5 \mathrm{~km}$ [2]. Since then, wireless communications has been developed at an impressive rate. In 1941 the US Federal Communications Commission (US FCC) licensed TV broadcasting. In 1981, the first generation of mobile phones was introduced and "IEEE 802.11", the current wireless LAN standard, was first released in 1997.

As a result of this rapid development, current radio engineers have to face a new issue: the frequency band most suitable for wireless communication was almost fully allocated; there is not much new bandwidth available for additional wireless services but the demand for further expansion of wireless communications services continues [3].

However, even though the spectrum is almost fully allocated, it is not fully used [4]. Henceforth, reusage of the frequency spectrum was recognised as an opportunity to break the spectrum gridlock. Two approaches have emerged and attracted a lot of attention: Cognitive Radio and Ultrawideband. Cognitive radio uses active spectrum sensing to detect unused bands in the spectrum. If such band is detected, it can be utilised for communication. Therefore, no interference to the primary spectrum users appears. Ultrawideband, on the other hand, re-uses the spectrum elegantly by transmitting an extremely wideband signal $(\mathrm{B}>500 \mathrm{MHz}$ ) with a low power spectral density. For the primary licensed narrowband users, a UWB transmission appears as white noise. Conversely, the narrowband user represents only narrowband interference to the UWB system and this interference 
can be resolved using the inherent frequency diversity of the UWB system as long as the front end of the UWB receiver is not overloaded.

These two concepts are not competing with each other. In fact they are complementary. The range of ultrawideband is limited due to the low power spectral density to approximately $10 \mathrm{~m}$ [5]. Thus it allows extremely dense frequency re-usage. Also, due to its immense bandwidth it offers extremely high available channel capacity even for low Signal-to-Noise Ratio (SNR). As a result, it is the candidate for short-range high speed wireless networks (data-rates in the magnitude of hundreds or thousands of Mbps). Cognitive radio, does not offer sufficient bandwidth to compete with UWB in the field of data-rate, but it outperforms UWB in the range. Hence, it is the candidate for wireless services over longer distance, e.g. future generations of mobile phones.

\subsection{History of UWB beginnings}

The first paper (apart from the military projects from 1960s [6]) to use the term ultra-wideband was published in 1991 by Fullerton [7]. However, according to IEEExplore, there have been only 35 papers on the topic in 1991-97. The real breakthrough started in 1998 when Win and Scholtz showed that time hopping codes enable the avoidance of catastrophic collision of multiple users using impulse radio communication [8]. The interest raised by reference [8] has further been fuelled by part 15 ruling of the US Federal Communication Commission in 2002 that has allowed unlicensed UWB radiation in the frequency band $3.1-10.6 \mathrm{GHz}$.

After this ruling, the interest of academia and industry has exploded. In the first years after the US FCC part 15 ruling, UWB was treated as the new wonder child in the world of wireless communication. It was "the technology" that was about to change the world. In 2004, Parks Associates Research Consultants estimated that the market for ultrawideband chips and gear will top $\$ 1,000,000,000$ by 2008 [9]. In the same year the TIME magazine selected UWB as one of the technologies to remember for 2005 [10].

It is not surprising that the standardisation process started very soon after the part 15 ruling. For the streaming data application the IEEE 802.15.3a task group was created in 2002. From several PHY alternatives, two proposals, both backed by strong industrial alliances, were selected. These approaches were Direct Sequence CDMA (DS-CDMA) supported by the alliance called the UWB Forum, and Multi Band OFDM (MB-OFDM) which was the proposal of the WiMedia alliance [11]. Both alliances never managed to reach a compromise, and as a result the 802.15.3a task group ceased its existence in early 2006 and each of the Alliances was expected to create their own standards and let the marketplace to make the decision [11].

The WiMedia alliance finalised their proposal and submitted it to ECMA (European association for standardising information and communication). The PHY and MAC specifications were incorporated 
in the ECMA-368 document [11]. Meanwhile, Motorola's spin-off, Freescale, abandoned the UWB Forum. Soon after, other members started leaving the body [11]. At the present time, the UWB Forum is effectively lifeless.

With this development, it seemed as if the battle had been decided even before the technology entered the marketplace and the WiMedia alliance seemed the like winner. However, this was not exactly the case. It turned out that the selected architecture of UWB system was too complex to produce chips at a cost that would lead to a breakthrough in the marketplace [12]. According to [12], the chip cost was originally $\$ 15$ and has now been reduced $\$ 6$, which is a significant improvement. Reference [12] also draws a parallel with Bluetooth devices and claims that the cost should fall below $\$ 5$ to make the necessary market breakthrough.

The cost typically falls as the technology matures it seemed to be only a matter of time until UWB achieves it prophesied market position. Unfortunately, like other industries even UWB was affected by the financial crisis in 2008. As a result, in March 2009 the WiMedia alliance announced the transfer of its specifications to two partner groups (USB Implementors Forum and Bluetooth SIG) and published plans to disband [12]. A month later (April 2009) another UWB start up, Radiospire, that used the OFDM approach announced bankruptcy which prompted commentators to go as far to claim that "UWB is dead in consumer electronics..." [13].

\subsection{Where is UWB now?}

The events described by references $[12,13]$ might indicate that one should ask whether the days of UWB are over. However, the on-going interest of academia and companies like Samsung, Tialinx, Inc., Wisair and NetGear in the UWB technology shows that statements like "UWB is dead in consumer electronics..." [13] are far from true. It is true that the development of market-ready products took longer than expected but this is due to the fact that UWB is fundamentally different than standard narrow- or wideband-communication rather than due to the fact that the potential of the technology has been overrated in the early 2000s. As a result, numerous challenges had to be overcome which caused delays not anticipated in 2002.

Whilst these delays in combination of the market meltdown in 2008-2009 might have lead to withdrawal of some investors, they did not remove the pressing issue of full spectrum allocation as outlined in Section 2.1. Furthermore, one of the biggest achievements of the technology is the fact that regulatory bodies worldwide have approved UWB radiations which effectively enables the reusage of spectrum by UWB devices. The current regulatory standards are summarised in section 3 because they define framework that limits the potential capacity, range or the way multiple antenna systems can operate.

\section{Worldwide regulatory standards}


UWB has been assessed by the regulatory bodies worldwide, and whilst there are superficial differences among their rulings, UWB is generally approved in Europe, USA, Japan, South Korea and others. As a result, there are no legislative limitations and the UWB wireless devices may freely enter the markets. The regulations in Europe, South Korea and Japan changed recently and the current status is summarised in Fig. 1.

In Europe, the regulation has changed in 2009. Now it defines a 'general limit' that allows UWB transmission. The general limit applies to any device indoors or for indeed for non-fixed including those outdoors. The 'general limit' allows use of the frequency range of $6-8.5 \mathrm{GHz}$ with an average Equivalent Isotropic Radiated Power (EIRP) spectral density of $-41.3 \mathrm{dBm} / \mathrm{MHz}$ [14]. The regulation also allows emissions in the $3.1-4.8 \mathrm{GHz}$ band with mean EIRP density of $-41.3 \mathrm{dBm} / \mathrm{MHz}$ but only under the assumption that Low Duty Cycle (LDC) mitigation techniques will be used. This regulation specifies that the sum of all signals is less than $5 \%$ of the time averaged over each second and less than $0.5 \%$ of the time averaged over each hour and no transmitted signal can last longer than $5 \mathrm{~ms}$ [14]. Alternatively, if Detect and Avoid (DAA) is employed, a mean EIRP density of $-41.3 \mathrm{dBm} / \mathrm{MHz}$ is allowed (on the top of the general limit) in the bands of $3.1-4.8 \mathrm{GHz}$ and $8.5-9.0 \mathrm{GHz}$ [14]. The objective of DAA is to avoid a frequency band if a device can detect spectrum used by other service. The details of the European requirements on DAA can be found in [15] or in the ETSI norm EN 302 065.

The regulations in the USA are the most liberal compared to other areas. UWB radiation for communication is allowed in the band $3.1-10.6 \mathrm{GHz}$ with an average EIRP density $-41.3 \mathrm{dBm} / \mathrm{MHz}$ [16]. The rules for out-of-band radiation are stricter for outdoor emissions. Transmission at low frequencies is allowed for applications such as ground penetrating radar.

Japan, in contrast, has strict rules. UWB is allowed indoors only and it is specified that there has to be a mains powered 'host' device and a battery powered 'client' [11]. This sets additional constraints on power consumption of UWB devices. In terms of frequency spectra, the allocated frequency spectrum is $7.25-10.25 \mathrm{GHz}$ at a mean EIRP density of $-41.3 \mathrm{dBm} / \mathrm{MHz}$. The $3.4-4.8 \mathrm{GHz}$ band is allowed for devices using DAA technique.

The regulation in South Korea allows indoor and outdoor operation. The approved frequency bands are similar to the ones in Japan. Mean EIRP density of $-41.3 \mathrm{dBm} / \mathrm{MHz}$ is allowed in the band $7.2-$ 10.2 GHz. The band 3.1 - 4.8 GHz with mean EIRP density of $-41.3 \mathrm{dBm} / \mathrm{MHz}$ can be used if DAA is employed.

\section{Current challenges for UWB}

Unfortunately, despite the positive outlook for the UWB there are still some issues that cause the gap between the theoretical potential of the UWB technology and practical performance achievable 
by market-ready products. These issues will be presented in this section whilst various approaches to alleviate them will be discussed in section 5 .

The data-rate, reliability and range of UWB are issues standard for most wireless communication systems and similarly to other systems, even in UWB an improvement on all three fronts is being sought.

In terms of data-rate, the ECMA WiMedia standard supports 8 data-rates ranging from $53.3 \mathrm{Mbps}$ to $480 \mathrm{Mbps}$, but the initial expectations for UWB were in the area of Gbps. Therefore, ways to increase these data-rates are being explored.

In terms of reliability, if UWB intends to replace wired data links, it has to provide the same guarantee for quality of service. Current systems in real scenarios often perform below the specifications. For instance, ECMA Wimedia device with multiple-antenna receiver tested in [17] failed to provide throughput above $250 \mathrm{Mbps}$ for line-of-sight scenarios and ranges below $6 \mathrm{~m}$ even though according to the standard specifications a throughput of $320 \mathrm{Mbps}$ should not represent any challenge even for a single antenna device [11].

The range of the UWB system is strictly limited by the regulated EIRP of $-41.3 \mathrm{dBm} / \mathrm{MHz}$ and by the noise floor at $-114 \mathrm{dBm} / \mathrm{MHz}$. These boundaries represent a very strict framework but allow us a range estimation for a chosen modulation scheme using a link budget analysis. However, similarly to data-rate consumers are always hungry to expand the range.

Chip complexity has already been mentioned as one of the main reasons for the delay in introducing Wimedia devices to the market. Even though the chip cost decreases as the technology matures simply with the production volume. It must be noted that complexity reduction still needs to be considered especially as it increases in relation to improvements in reliability (e.g. more complex coding), range (e.g. signal processing in MIMO systems), data-rate (higher data-rate requires faster clock) or the plans for future generation including WiMedia systems doubling the bandwidth or 60 GHz systems relying on OFDM with 512 subcarriers (4 times more than current WiMedia standard). Another issue is the inherent challenge of frequency re-use. This includes robustness against the presence of a narrowband interferer especially for the cases of strong interferers that might overload the front-end of the receiver. This issue includes two tasks: detection, and avoidance or suppression. An implementation of detect-and-avoid algorithms is also of interest as it enables the access of additional bandwidth in Europe, Japan and Korea as presented in Section 3.

\section{Current Research Topics}

Today's challenges for UWB technology have been presented in Section 4. They are chip complexity, data-rate, reliability, range, interference by narrowband systems in proximity. These issues are often inter-related. Therefore for the sake of clarity, this section does not assign research topics to the 
individual issues. Instead, the research topics are grouped and described in six subsets. For each subset it is explained which issues are addressed and how.

\subsection{Chip Complexity of OFDM Systems}

The chip complexity/cost of OFDM systems is caused mainly by the complexity of the Fast-FourierTransform employed by OFDM. The least computationally demanding FFT implementation known to the authors is presented in [18]. According to [17] the number of FLOP counts for a FFT with length $\mathrm{N}$ is [17]:

FLOPs $=\frac{34}{9} N \log _{2} N-\frac{124}{27} N-2 \log _{2} N-\frac{2}{9}(-1)^{\log _{2} N} \log _{2} N+\frac{16}{27}(-1)^{\log _{2} N}+8$

With the ECMA-368 specification of a WiMedia OFDM symbol length of 312.5 ns [11], the FFT with length 128 requires $8.934 \cdot 10^{9} \mathrm{FLOP}$ counts per second. This is not the full load of the chip as it also needs to perform other operations such as forward error correction. Ideally, all the arithmetic operations should be performed by a chip suitable for handheld devices. To compare, this FFT computational complexity is approximately 25 times higher than required by chips for $802.11 \mathrm{a} / \mathrm{g} / \mathrm{n}$ WiFi. This complexity translates into chip cost.

Whilst it is true that chip cost falls down as the technology matures, the wait is a test of customer's and investor's patience. Hence, research investigating options to reduce the chip complexity of UWB OFDM based system remains essential due to the non-linearity of Eq. (1). Resulting from (1), the WiMedia 480 Mbps offered by Wimedia is 8.89 -times more than 54 Mbps of $802.11 \mathrm{a} / \mathrm{g}$ but at the cost of 25-times higher FLOP count. Thus, increase in data-rate by scaling the OFDM represents an additional challenge in terms of chip complexity and should enter consideration about for future systems such as the next generation of WiMedia systems with OFDM symbol bandwidth of 1056 $\mathrm{MHz}$ providing data-rates in magnitude of $1 \mathrm{Gbps}$ [11] or future $60 \mathrm{GHz}$ systems.

Consequently, one of the main research topics in the current UWB landscape is chip optimisation and low complexity chip design for WiMedia OFDM that would enable commercially viable operation. The related works are references [19-23] and references therein.

\subsection{Non-OFDM approaches}

In terms of reliability, OFDM without channel coding and frequency diversity schemes does not provide the most reliable implementation of UWB system as it capitulates on the exploitation of frequency diversity of UWB systems. To be more specific, individual narrowband subcarriers in the OFDM symbols suffer from frequency selective fading in the wireless channel. In the ECMA-368 WiMedia specification [11], if only one of the subcarriers suffers from strong fading its individual biterror-rate (BER) will achieve the maximum value of 0.5 and even if all other subcarriers are transmitted error-free the overall BER of the gross data stream will yield $4 \cdot 10^{-3}(=1 / 256)$. This number is increased $4 \cdot 10^{-3}$ for each additional subcarrier that suffers multipath fading. This might 
be alleviated by channel coding but due to packet length even small BER results in substantial Packet Error Rate (BER of $5 \cdot 10^{-5}$ yields in PER of 33.6\%). This is seen as one of the reasons for performance reported in [17] and discussed in Section 4. This substantiates the implementation of robust protection by means of frequency diversity and forward error correction [11]. These measures present additional computational load for the chip whilst sacrificing the data-rate. In WiMedia systems, the gross data-rate of $640 \mathrm{Mbps}$ is scaled up to $53.3 \mathrm{Mbps}$ in the basic mode with strongest channel coding and as noted in Section 5.1, the 802.11a/g standards can offer 54 Mbps with chips performing FFT requiring 25 -times less FLOP/s.

On the other, it has been well proven that as the signal bandwidth increases, the individual multipaths can be resolved and the channel energy does not change for small displacements of the transmitter or receiver [24-27].

At a glance, this would lead to the return to impulse radio as envisaged by the initial paper from Win and Scholtz [8], which would not lead to the most practical solution as impulse radio with GHz pulses suffers from issues such as complex rake receivers [28], clock synchronisation, complex pulse generation etc. [5,6]. However, other implementations using wideband carriers such as Single Carrier UWB with Frequency Domain Equalization (SC-FDE) [28] or Multi-Tone Frequency Shift Keying (MTFSK) [29] have been studied. These modulation schemes are introduced in Sections 5.2.2 and 5.2.3 respectively. Section 5.2.1 summarises the knowledge about frequency selective fading in UWB wireless channels because its conclusions are crucial for the SC-FDE and MTFSK UWB wireless systems.

\subsubsection{Frequency Selective Fading}

A recent theoretical study [30] of the frequency selectivity shows that the bandwidth required to resolve individual multipaths depends on the volume of the room and from its conclusion it is shown that the bandwidth required to resolve individual multipaths in a typical indoor environment is in the order of $200 \mathrm{MHz}$. For the sake of clarity the main point of [30] is repeated here. Reference [30] starts with the Saleh-Valenzuela model of channel impulse response [30]:

$h(\tau)=\sum_{l=1}^{L_{c}} \sum_{k=1}^{L_{r}} \alpha_{k, l} e^{j \varphi_{k, l}} \delta\left(\tau-T_{l}-\tau_{k, l}\right)$

where $h(\tau)$ is the channel impulse response, $\alpha_{k, 1}$ and $\varphi_{k, l}$ represent the multipath gain and phase coefficient of the $\mathrm{k}^{\text {th }}$ ray in $\mathrm{l}^{\text {th }}$ cluster, $\mathrm{T}_{\mathrm{l}}$ stands for the time-of-arrival of $\mathrm{l}^{\text {th }}$ cluster, $\tau_{\mathrm{k}, \mathrm{l}}$ is ray arrival time within individual cluster, $\delta(\mathrm{t})$ is Dirac impulse, $L_{r}$ and $L_{c}$ define the total number of rays/clusters.

The Fourier transform of Eq. (2) is then used to determine the energy of the channel transfer function for a centre frequency, $f_{0}$, and bandwidth $B W$, after some mathematical operations we obtain [30]: 
$\int_{f_{0}-B W / 2}^{f_{0}+B W / 2}|H(f)|^{2} d f=$

$B W\left(\sum_{l=1}^{L_{c}} \sum_{k=1}^{L_{r}} \alpha_{k, l}^{2}+\right.$

$\left.\sum_{l=1}^{L_{c}} \sum_{\substack{n=1 \\\{l, k\} \neq}}^{L_{c}} \sum_{\substack{k=1 \\\{n, m\}}}^{L_{r}} \sum_{m=1}^{L_{r}} \alpha_{k, l} \alpha_{m, n} \operatorname{sinc}\left(\pi B W B_{l, n}^{k, m}\right) \cos \left(2 \pi f_{0} B_{l, n}^{k, m}+\varphi_{m, n}-\varphi_{k, l}\right)\right)$

where $B_{l, n}^{k, m}=\left(T_{l}+\tau_{k, l}\right)-\left(T_{n}+\tau_{m, n}\right)$ is the time difference between rays from different clusters , and $\{k, l\} \neq\{n, m\}$ represent the summation condition $l \neq n \quad O R k \neq m$

Analysis of Eq. (3) provides insights into the nature of the fading. The final expression consists of two terms. The double sum represents the total energy carried by the rays, the quadruple sum represents the impact of fading due to the superposition of pulses. The impact of bandwidth can be observed in the term $\operatorname{sinc}\left(\pi B W B_{l, n}^{k, m}\right)$. For a wideband channel with $B W \rightarrow \infty$, the quadruple summation term disappears and the channel does not manifest any frequency selectivity. For $B W \rightarrow 0$, the term $\operatorname{sinc}\left(\pi B W B_{l, n}^{k, m}\right) \rightarrow 1$. The energy of the channel transfer function then depends strongly on the magnitude and polarity of $\alpha_{k, l}, \alpha_{m, n}$ and $\cos \left(2 \pi f_{0} B_{l, n}^{k, m}+\varphi_{m, n}-\varphi_{k, l}\right)$. For ECMA-368 WiMedia OFDM with 128 subcarriers spaced by $4.125 \mathrm{MHz}$, the $\alpha_{k, l}, \alpha_{m, n}$ are approximately same for all subcarriers, but the $\cos \left(2 \pi f_{0} B_{l, n}^{k, m}+\varphi_{m, n}-\varphi_{k, l}\right)$ term will change significantly. For example, for $B_{l, n}^{k, m}=10 \mathrm{~ns}$ (this is a realistic value for an indoor environment as it corresponds to a multipath path-length difference of $3 \mathrm{~m}$ ), the frequency sweep over the bandwidth of $528 \mathrm{MHz}$ (over all 128 subcarriers) equals to sweep over $10 \pi$ in the argument of the cos-term. As a result, some subcarriers that are amplified (cos-term positive) whilst others that are attenuated (cos-term negative).

For the symbols with bandwidths of $200 \mathrm{MHz}$, the $\operatorname{sinc}\left(\pi B W B_{l, n}^{k, m}\right)$ is plotted in Fig. 2 to against $B_{l, n}^{k, m}$. From Fig. 2, it can be read that for $B_{l, n}^{k, m}>4 \mathrm{~ns}$, the value of the $\operatorname{sinc}\left(\pi B W B_{l, n}^{k, m}\right)$ will be below $-6 \mathrm{~dB}$. Under the reasonable assumption $\left|\alpha_{k, l}^{2}\right|>\left|\alpha_{k, l} \alpha_{m, n}\right|$ as later cluster suffers more attenuation due to larger path loss, it can be concluded that for environment where the delay between multipaths will be above 4 ns (1.2 meter path difference) the total erasure of the symbols with bandwidth of $200 \mathrm{MHz}$ will be extremely unlikely.

Noted that the delay among individual rays in the Saleh-Valenzuela model is often around $1 \mathrm{~ns}$ [6]. Whilst this is true, this applies only to rays within a cluster in Eq. (2). The rays from one cluster do are not independent. References [31,32] have conclusively proven that the clusters are a manifestation of antenna impulse response. Thus, these rays are deterministic and any frequency selectivity associated with them is given by the antenna and it does not change for small displacements of the transmitter or receiver [32]. Furthermore, deep radiation minima of UWB 
antennas can be found outside the intended radiation, because UWB antennas are designed to radiate within the entire UWB frequency band.

To further support the claim that a symbol with bandwidths of $200 \mathrm{MHz}$ are sufficiently robust against fading in indoor environment, reference [33] analysed the change of the parameters of the Nakagami probability density function (pdf) describing the statistics of spatial fading for different channel bandwidths using the experimental data from [27]. This change in the parameters is best documented by Fig. 3, presenting the pdf shape for four different bandwidths for centre frequency of $6 \mathrm{GHz}$. For the bandwidth of interest, $200 \mathrm{MHz}$, only in $0.4 \%$ of all cases will the signal experience fading below $-6 \mathrm{~dB}$. In other words, a total erasure of the signal is almost impossible.

To conclude, this section has presented a theoretical insight into the causes for frequency selectivity in wireless channels. It has shown that even signals with bandwidths of $200 \mathrm{MHz}$ are robust against the total erasure due to multipath propagation. This finding can be used in the synthesis of alternative modulation schemes robust against frequency selectivity of wireless channel that require weaker compared to the ECMA WiMedia OFDM.

\subsubsection{Single Carrier UWB with Frequency Domain Equalization}

The idea of Single Carrier Frequency Domain Equalisation (SC FDE) is almost three decades old and has been introduced for the first time in 1973 [34]. Later, was studied as an alternative to OFDM for wideband wireless systems $[35,36]$ and was introduced for UWB for the first time in reference [28], the concept gained significant attention of the academic and was further developed in works [3741].

SC FDE UWB transmitters use wideband BPSK modulation of a single carrier, the pulses have bandwidth comparable to an entire OFDM symbols. Therefore, the pulses can capitalise on the frequency diversity as described in Section 5.2.1. In a strong multipath environment should attempt to use an equalizer that sums all the delayed copies of the signal corresponding. Time based systems use transversal filters with delay tap lines that are almost identical to rake receivers as utilised by Impulse Radio UWB [6]. However, the equalisation in time domain using delay tap lines proves to be extremely computationally complex [35] and unsuitable for real systems. Therefore, it is replaced by equalisation in frequency domain, which is fully equivalent to the time domain equalisation but less computationally complex [35].

In the frequency domain equalisation, data transmitted in blocks $\underset{x}{\rightarrow}$ are processed by FFT at the receiver. A channel estimate is used to perform equalisation on the $\vec{X}$, which is Fourier transform of $\vec{x}$, by multiplication with a complex vector $\underset{c}{\vec{C}}[34]$. Afterwards, the IFFT is applied to obtain back the time domain signal. This corresponds to the time domain equalisation where the copies of the signal are scaled by vector $\overrightarrow{c^{\prime}}$ and summed. 
The obvious issue is the fact that due to multipaths, the blocks at the receiver will overlap. This is solved by the use of cyclic prefix long enough to prevent inter-block-interference $[28,34,35]$.

The SC FDE receiver is more complex than its OFDM counterpart as it performs both FFT and IFFT but there is no FFT at the transmitter. Hence in terms of computational complexity of the FFT, this modulation scheme is fully equivalent to OFDM [35], but it is more robust against frequency selective fading and without channel coding, it significantly outperforms its OFDM equivalent [28], reducing the commitment of computational assets for channel coding and de-coding.

Furthermore, the signal's peak to average ratio is reported to be lower than in the case of equivalent OFDM symbol. This reduces the required linearity range of power amplifier at the transmitter which can be translated in further cost reduction of the final chip $[28,35]$.

Lastly, as shown in $[35,36]$ the SC FDE scheme is in many ways similar to a OFDM receive, only the IFFT is performed at different stages of the communication chain. SC FDE can therefore co-exist and and share the resources with OFDM within one system [35].

The concept of SC FDE UWB also seems to have entered the commercial arena. Pulse Links' CWave Wireless solution [42] uses BPSK at a signalling rate of $1.3 \mathrm{GHz}$ and carrier centre frequency of $4 \mathrm{GHz}$ [42]. The output is filtered to fulfil the regulatory requirements. The equalisation at the receiver is not described but it SC FDE seems to be the most-likely candidate. The system is reported to be capable of data rates above $320 \mathrm{Mbps}$ for range below $10 \mathrm{~m}$ [42]. This system has two main issues. It is not compliant with the new European where the unlicensed UWB spectrum is in the $6.0-8.5 \mathrm{GHz}$ band. Neither is it compliant with Japanese or South Korean regulations. Further, it is not capable of multiple user access, but the possibility of multiple user access schemes for SC FDE systems has already been investigated in [38]. Lastly, co-existence with other UWB systems using different modulation schemes has to be investigated.

\subsubsection{Multi-tone FSK}

Another approach to utilise fade depth scaling with bandwidth (introduced in section 2.3.1) in design of more efficient UWB systems has been introduced recently in reference [29], which proposes a Multitone Frequency Shift Keying (MTFSK) as first investigated by a theoretical study in [43] and [44]. MTFSK is similar to standard FSK, but it expands the available alphabet by sending more tones at a time.

Reference [29] focuses on tone bandwidth optimisation of the concept towards UWB. It shows that the bandwidth of tones can be optimised to make use of the fact that wideband signals are more robust against the impact of fading (Section 5.2.1). Empirical simulations show that the fading can be neglected for tone bandwidths above $100 \mathrm{MHz}$. This significantly reduces the computational power 
that has to be allocated to process the redundancy that has to be present in OFDM systems [11]. An increase of bandwidth above $500 \mathrm{MHz}$ is not recommended by reference [29] because longer pulses are more robust against small displacement in terms of synchronisation.

Reference [29] also notes that due to inter-symbol-interference, which occurs for shorter symbols [11], it is necessary to employ a guard interval and so to reduce the duty cycle of the system. It is also shown that, overall, this approach is advantageous. Firstly, the number of tones is reduced and low complexity analogue processing is possible, which significantly reduces the computational requirements on the chip (recall $8.934 \cdot 10^{9} \mathrm{FLOP}$ counts per second for MB-OFDM). Each subcarrier is processed by an analogue filter. For a low number of subcarriers, this is not a constraint for implementation and corresponds to a chip performing digital FFT at rates described in Section 5.1.

Secondly, it is shown that there is a trade-off between low duty cycle and the complexity of forward error correction codes. In other words, the chip computational load can be traded for spectral efficiency. Whilst spectral efficiency is the holy grail of mobile network design, it is less relevant in UWB where enormous bandwidths are available.

In no way are the authors suggesting that spectral efficiency is completely irrelevant. In this aspect, UWB can be compared to mobile phones. 2G GSM transmission have link spectral efficiency of 0.52 $\mathrm{bit} / \mathrm{s} / \mathrm{Hz}$. The latest 3.9G LTE transmissions can achieve up to $16.32 \mathrm{bit} / \mathrm{s} / \mathrm{Hz}$. This improvement has been introduced within a time frame of 18 years and it would have never been achieved without the phenomenal market success of 2 G GSM. 18 years is an extremely long period bearing in mind that the first wireless transmission over a longer distance was presented 116 years ago. In other words, whilst the spectral efficiency of UWB should not be crucial at the moment, there is no doubt that in the long run UWB communications will have to start increasing spectral efficiency to satisfy the ever increasing hunger for wireless streaming media.

\subsubsection{Impulse Radio}

Another non-OFDM based concepts for UWB are the original impulse radios as suggested by the original UWB works from the 1990s e.g. $[7,8]$. There has been a substantial amount of research into Impulse Radio UWB (IR UWB). For instance, at the time of writing this article, the search for "IR UWB" in IEEExplore $^{\mathrm{TM}}$ returns 750 conference papers and 175 journal papers.

The main idea of impulse radio is the use of extremely short pulses (sub-nanoseconds to nanoseconds) with low duty cycle. These pulses are shorter than the distance between multipath components in the channel impulse response, thus individual multipaths can be resolved and no frequency selective fading occurs. The guard interval between pulses is chosen to prevent inter- 
symbol-interference for typical delay spreads of channel impulse response in indoor environments. There is a plethora of modulation schemes utilised in IR UWB including e.g. Frequency Shift On-Off Keying (FSK-OOK), Pulse-Amplitude-Modulation (PAM), Pulse-Position-Modulation (PPM), where data is coded by relative position of the pulse in symbol [45].

However, despite the intensive research, Impulse Radio still faces significant challenges with the complexity of the receiver [28] and performance of real system implementations is significantly inferior to the performance of commercial WiMedia systems. This can be illustrated by recent work [46], where a state-of-the-art Non-Coherent FSK-OOK Impulse Radio demonstrator can only provide data-rate of $30 \mathrm{Mbps}$ over a distance of $30 \mathrm{~cm}$ with BER of $10^{-2}$. WiMedia OFDM systems can provide hundreds of Mbps over a distance of few meters as shown in [17]. From this point of view, the IR seems to be the least mature technology. On the other hand, there is still a significant potential for it. Recalling the European Regulations (Section 3), a significantly larger bandwidth can be used by systems with Low-Duty-Cycle (LDC). Impulse radio is an ideal candidate for LDC applications with lower-data-rate such as sensor networks. Whilst, the data-rates of few Mbps can be achieved by other technologies such as Bluetooth, the advantage of UWB is the possibility of denser spectral reuse and with the use of Time-Hopping Multiple Access Schemes [8] the possibility to accommodate more users without strong interference.

In nutshell, even though practical IR UWB systems do not currently offer data-rates and range comparable to WiMedia OFDM systems, it remains highly attractive for certain type of applications especially considering the regulatory frameworks in some parts of the world. Therefore, this section will focus on discussion of the main challenges IR UWB currently faces.

The first issue is the choice of Coherent/Non-coherent receiver architecture. Coherent receivers correlates the received signals with a carefully-designed template signal. It outperforms noncoherent receivers in minimizing BER for AWGN channel without inter-symbol interference. In [46] it is shown that for perfect synchronisation the gap between coherent and non-coherent in UWB systems is $9 \mathrm{~dB}$ which is "more than the $3 \mathrm{~dB}$ gap known from narrowband systems" [46]. This seems to be a huge drawback of non-coherent receivers because the $9 \mathrm{~dB}$ penalty in SNR is extremely painful in the UWB landscape where the available dynamic range between the limited EIRP and the noise floor. For UWB systems, this dynamic range is $72.7 \mathrm{dBm}$ (=EIRP (-41.3 dBm/MHz) - noise floor $(-114 \mathrm{dBm} / \mathrm{MHz}))$ which is further reduced by mean path loss (approx. $45 \mathrm{~dB}$ for a distance of $1 \mathrm{~m}$ e.g. [6]) and noise figure of the receiver (realistic assumption of 4-5 dB [5])

On the other hand, the correlation receiver is associated with extremely high implementation complexity because it requires almost perfect synchronisation [47]. Consequently, it requires precise and complex channel estimation [48] which is not suitable for low-complexity low-cost applications, 
the target marketplace for IR UWB. Furthermore, to capture the energy spreaded into many multipaths, coherent receivers have to employ the rake architecture with many fingers. This is further increases the complexity especially high sampling rates of ADCs is required [28]. Some of these issues might be alleviated by future research. For instance, references $[31,32]$ have shown that rays within a cluster of Saleh-Valenzuela model e.g. $[6,30]$ are a deterministic manifestation of antenna impulse response and the waveforms of these manifestations do not change for small displacement [32]. This may potentially simplify the channel estimation problem as well as reduce the number of fingers in rake receiver.

Non-coherent receivers outweigh their performance degradation by the use of low complexity architecture. Firstly, they are more robust against synchronization errors and do not require complex channel estimation $[46,49,50]$. Furthermore, they do not need the rake structure $[46,49,50]$ and "only frame or symbol rate sampling" [50]. Two designs most studied in the literature are the energy detectors and autocorrelation receivers. The energy detector has mainly been considered for FSKOOK systems as it can be implemented as a low complexity filter bank [49]. For PPM systems, the autocorrelation receivers are the suitable choice. The autocorrelation receives use the autocorrelation of the received signal. For the sake of brevity, the reader interested in details on the main schemes of autocorrelation receivers (transmitted reference and differential schemes), their performance and challenges for synchronisation is referred to works $[46,50]$ and references therein. To conclude this section, impulse radio remains in the focus of researchers due to its potential for low data-rate low-complexity applications such as sensor networks. There are two schemes coherent and non-coherent receivers. The main challenge for coherent receivers is the development of lowcost low-complexity channel estimators for precise synchronisation, while the main challenge for non-coherent receiver is the improvement of BER performance so that the system can provide acceptable BER within the limited dynamic range available for UWB

\subsubsection{Non-OFDM Modulation Schemes Conclusion}

This section has presented three concepts that use wideband signals which are robust with respect frequency selective fading in wireless channels. In the case of SC-FDE UWB and MTFSK UWB the requirements on the strength of channel coding and/or other requirements on the system such as the linearity of transmitter are reduced. As a result, such systems may offer same data-rate with lower complexity or a higher data-rate whilst maintaining the complexity. The IR UWB systems also do not suffer from frequency selective fading as all multipaths can be resolved. Even though the requirement for guard intervals between consecutive pulses does not make them suitable candidates for high-data-rate applications, this concept remains highly attractive for low-complexity 
low-cost low-data-rate applications such e.g. in sensor networks mainly due to the higher density of frequency re-use compared to other systems such as Bluetooth.

By no means does this section try to list all non-OFDM approaches. For the sake of brevity, it lists the schemes that the authors believe have the most potential for the future. For instance the direct spreading as proposed by UWB Forum to the IEEE (Section 2.2) is not discussed here even though there is still on-going academic research into the topic, e.g. [51-54].

\subsection{Narrowband Interference in UWB}

One of the issues that arise from the frequency re-use by UWB system is the interference between primary narrowband users and the UWB users. As mentioned in Section 2.1, the interference to primary users by UWB systems should be negligible because of the low EIRP spectral densities allowed by regulatory authorities. The situation of a narrowband interferer is different. There are generally two cases: a distant (weak) narrowband interferer and a close (strong) narrowband interferer.

The former situation is of a less concern. Such interferer reduces the SNR in a narrow frequency band. Effectively, it is similar to a deep frequency fade as described in section 5.2.1. Such interference should not significantly influence the performance of UWB systems due to their inherent frequency diversity. In OFDM systems the interference will cause a BER increase for one of the subcarriers but channel coding and frequency spreading schemes as implemented by the WiMedia OFDM systems can protect the total data-stream. In other non-OFDM systems such as SC FDE UWB, MTFSK UWB or IR UWB, weak narrowband interference will have little influence on the performance as the systems are inherently robust to a narrowband fading (See section 5.2).

However, the latter scenario of a strong interferer in the proximity of a receiver represents a more serious challenge and means to mitigate this problem enable to achieve lower BER or higher datarates. The biggest issue is the dynamic range of the receiver that is chosen appropriately to the expected power levels of UWB signals. A strong narrowband interferer in proximity may well be outside of this dynamic range and overload the front end of the receiver. Because UWB systems must be capable of operation even under such circumstances, various solutions are considered. The main approaches are: detect and avoid, detect and suppress, suppress

The ECMA WiMedia OFDM standard, for instance, provides means to detect such situation. The PHY layer estimates the total received RF power and the SNR of subcarriers [11]. For the case without an interferer, high received RF power is linked to high SNR estimate. However, in case of a strong 
interferer the SNR estimate is low despite high received RF power. This situation can be detected and a different band can be chosen.

The use of Detect and Avoid Algorithms (DAA) in UWB systems is of interest as DAAs are key to switchable band notch systems at the receiver as well as at the transceiver (described below). Furthermore, they are gaining an increasing interest, e.g. [58-61]., mainly due to the recent rulings of regulatory authorities in Europe, Japan and Korea that assign some frequency bands only to users with DAA to protect primary users such as WiMax (Section 3) and the attractiveness of additional bandwidth.

An alternative is the use of band notch antennas that of suppress the typical narrowband interferers such as the WiMax band (3.3-3.6 GHz) or the WLAN bands $(5.15-5.35 \mathrm{GHz}$ resp. $5.725-5.825 \mathrm{GHz}$ ) while efficiently covering the remainder of the UWB band $[55,56]$. This approach blindly suppresses the interferer without being aware of its presence. Band notch antennas can also be used in transmitters to reduce interference to primary users.

An alternative to blind suppression is the use of switchable band notch filters or antennas that switch the interferer band of only when a presence of a strong interferer is detected (e.g. by the means outlined in preceding paragraph) [57].

The last option that can be used to suppress the interferer is the use of null-steering at a multiple antenna receiver. The challenges and issues of multiple antenna systems for UWB are discussed in section 5.4 .

\subsection{Multiple Antenna Systems in UWB}

Multiple antenna systems are an attractive topic within the wireless community as the concept allows more efficient use of channel resources, interferer suppression or increased security $[62,63]$. The use of multiple antenna techniques in UWB offers the same benefits as in standard narrowband wireless and it helps to tackle some of the big challenges causing the gap between the potential and actual practical achievements of UWB. Namely it enables higher data-rates, and/or higher reliability (lower BER), and/or longer range, and/or better suppression of interferers [64,65]. According to [64], Single-Input-Multiple-Output (SIMO) systems can increase the spectral efficiency logarithmically and Mulitple-Input-Multiple-Output (MIMO) systems offer even linear increase.

Unfortunately, the framework of UWB is different for most narrowband multiple antenna systems, especially the $802.11 \mathrm{n}$ standard. The main contrast stems from the behaviour of antenna arrays for UWB bandwidth, the EIRP constrained regulations as opposed to power constrained regulations that apply e.g. for WiFi 802.11n. Therefore, this section is organised in three subsections dealing with: 
the impact of bandwidth on the array performance; multiple transmit antennas; and multiple receiver antennas.

\subsubsection{UWB Antenna arrays}

The extreme bandwidth available for UWB systems is a blessing and a curse at the same time. It brings a plethora of advantages such as inherent frequency diversity (which increases robustness against narrowband interference and frequency selective fading), enormous available data rate, availability of precise ranging etc. [6]. However, it is associated with many challenges as well, e.g. the design of antennas with frequency independent input impedance and radiation pattern, requirement of fast $A D C$ s to process the large bandwidth $[5,6,11]$. It is also an issue for a design of antenna arrays. The first issue with UWB antenna arrays is their description because using the title of [66] "it is a different world".

According to the literature, there are two basic ways to describe UWB array $[6,64]$ : the standard description as in narrowband systems by the means of array factor and antenna patterns or using the beam patterns that are summarised in $[64,66]$. Both descriptions have its advantages as well as limitations. The choice of the descriptor strongly depends on the choice of modulation scheme. The description by means of array factor is suitable OFDM modulations such as WiMedia (used e.g. in [65]), whilst beam patterns are more suitable for Impulse Radio systems [64,66].

\section{Array Factor as Descriptor for UWB Antennas}

It is well known from basic antenna theory that the array factor, $F_{A}$, for a uniform linear arrays along $y$-axis in the standard coordinate system can be calculated using following expression [6]:

$F_{A}=\left|\frac{\sin \left(\frac{M}{2}\left(\frac{2 \pi \cdot a}{\lambda} \sin \vartheta \sin \varphi+\psi\right)\right)}{\sin \left(\frac{1}{2}\left(\frac{2 \pi \cdot a}{\lambda} \sin \vartheta \sin \varphi+\psi\right)\right)}\right|$

Where $M$ represents the number of elements in the array, $\vartheta$ and $\varphi$ are standard polar coordinates, $\lambda$ is the free space wavelength, $a$ is the spacing of the arrays, $\psi$ is the phase difference between the elements in the array.

The array factor, $F_{A}$, strongly depends on the relative ratio of $\frac{a}{\lambda}$ which differs significantly between the lower and upper edge of the FCC UWB band (3.1 GHz resp. $10.6 \mathrm{GHz})$. As a result, the array factor can change significantly over the frequency band as illustrated in Fig. 4 showing example array factor for the lower and upper end of the FCC UWB spectra. The practical result of the change of the array factor with frequency is that the WiMedia OFDM systems will have to perform beam forming optimisation individually for each of the $528 \mathrm{MHz}$ band they use whilst performing the frequency hopping [11]. This figure also proves why array factor is a good descriptor for OFDM systems such as WiMedia and points out the issue of element spacing. 
In terms of suitability of array factor as descriptor for WiMedia OFDM, subplots a) and b) in Fig. 4 show the variation of the array factor for a frequency variation by $500 \mathrm{MHz}$ which corresponds to the bandwidth of OFDM symbols. The array factor is almost invariant for frequencies of a WiMedia OFDM symbol, thus it can be used as a descriptor. Furthermore, in OFDM the information is effectively transmitted in parallel by narrowband subcarriers, therefore the array processes narrowband signals rather than short wideband impulses. Therefore it seems appropriate to use the well developed narrowband framework.

Secondly, Fig. 4 shows the issue of element spacing. For the frequencies above $10 \mathrm{GHz}$, the spacing by $3 \mathrm{~cm}$ already fulfils the criteria for existence of grating lobes which can be identified in Fig. 4b. However, a straightforward reduction of the spacing is not always possible. Firstly, reduction of the element spacing negatively impacts the beam forming capabilities at the lower end of the spectra as the beam width will increase and the resulting array factor will resemble an isotropic radiator. Secondly, there are physical limits on the spacing of antenna elements because of the physical size of antennas which is proportional to a fraction of free-space wavelength at the lower end of the spectra [6].

In summary, this subsection has shown why the array factor ia a good descriptor for WiMedia OFDM systems. Its main advantage is that it enables access to an existing framework developed for narrowband systems (summarised e.g. in [62]). The issues associated with large bandwidth such are the element spacing and need of individual beam forming for each band used during frequency hopping.

\section{Beam Pattern Description for Impulse Radio}

For narrowband systems, the beamforming is performed by varying the phase between adjacent elements (and/or the amplitude weighting). However, the practical realisation of a phase shift corresponds to a time delay. For UWB impulses, the phase shift becomes ambiguous whilst the delay is precise and specific. Therefore, a set of new descriptors for UWB IR have been developed $[64,66]$. One of the main advantages of UWB beam formers is the non-existence of grating lobes $[64,66]$ because delayed signals add only in the direction of the two main lobes as can be read from equations presented later in this section.

These descriptors are introduced with the help of Fig. 5 that displays a uniform array consisting of $N$ omni-directional elements along the $y$-axis, spaced by element distance $d$, each receiving an incoming signal $s(t)$ incoming from angle $\alpha$. These signals are are weighted by coefficient $h_{i}$ and delayed by $\tau_{i}$ which are delays spaced by a constant step $\Delta \tau$. Using the notation from [64], the signals $y_{i}(t)$ can be described as:

$y_{i}(t)=s\left(t-\frac{d}{c} \sin \alpha\right)$ 
The total signal produced by the array can then be calculated as [64]:

$y(t)=\sum_{i=1}^{N} h_{i} y_{i}(t-n \Delta \tau)$

Where $\Delta \tau$ is a steering delay and for beamforming towards the direction of the incoming wave, it should be [64]:

$\Delta \tau=-\frac{d}{c} \sin \alpha$

The sign is chosen to correspond to Fig. 5.

Eqs. (5) - (7) are specific for a signal incoming from a $s(t)$ fixed angle $\alpha$, but they can be rewritten for a signal arriving from a general angle $(\vartheta, \varphi)$. Eq. (6) then turns into:

$y(t, \Delta \tau, \vartheta, \varphi)=\sum_{i=1}^{N} h_{i} s\left(t-\frac{d}{c} \sin \varphi-(n-1) \Delta \tau, \vartheta\right)$

Where $\Delta \tau$ is essential for the direction of the beam in horizontal plane. Elevation $\vartheta$ is not considered in this case as the array elements are assumed omni-directional and all are positioned in the horizontal plane.

Eq. (8) can then be used to calculate beam pattern. References $[64,66]$ then introduce three different ways to calculate beam patterns. These are introduced and discussed below $[64,66]$ :

$B P_{I}(\Delta \tau, \vartheta, \varphi)=\frac{\int_{-\infty}^{\infty}|y(t, \Delta \tau, \vartheta, \varphi)|^{2} d t}{\int_{-\infty}^{\infty}|s(t)|^{2} d t}$

$B P_{I I}(\Delta \tau, \vartheta, \varphi)=\frac{\max _{t}|y(t, \vartheta, \varphi)|^{2}}{\max _{t}|s(t)|^{2}}$

$B P_{I I I}(\Delta \tau, \vartheta, \varphi)=\frac{\max _{t}\left\{\int_{t-\frac{T}{2}}^{t+\frac{T}{2}}|y(t, \Delta \tau, \vartheta, \varphi)|^{2} d t\right\}}{\max _{t}\left\{\int_{t-\frac{T}{2}}^{t+\frac{T}{2}}|s(t)|^{2} d t\right\}}$

All three types of beam patterns compare the energy of the received signal to the energy of the transmitted UWB pulse $s(t)$. They differ in the integration time, this can be translated to the type of receiver that is expected to process the received signal $y(t)$. Whilst references $[64,66]$ discuss the impact of integration time in (9)-(11), they do not link them to different receiver architectures.

The first beam pattern $B P_{I}(9)$ considers the entire energy of the received signals. Thus it assumes situation of a receiver that captures the entire energy of all multipaths. It can be a coherent rake receiver with sufficient number of fingers, or non-coherent receiver (energy detector or autocorrelation detector) with sufficiently long guard intervals between symbols.

In practical realisations of receiver, the infinite integration will be replaced by integration over a finite time $T$. This corresponds to the last beam pattern $B P_{I I I}$. Thus, in agreement with [64] seems to be most suitable for description of actual systems. Especially for the case when there will not be sufficient resources at the receiver to process all the channel energy. For instance, each antenna receives $M$ multipaths and the $N$ element beamformer ideally adds only the first ray, signal $y(t)$ will 
manifest $1+N(M-1)$ multipath components which might be more than a coherent rake receiver with limited number of fingers can process.

If time $T$ is reduced to be shorter or equal to duration of symbol $s(t), B P_{I I I}$ is effectively turned into $B P_{I I}$, which corresponds to a receiver processing only one multipath. Whilst for single antenna systems such approach seems not-feasible because later multipath components carry significant amount of energy, in a $N$ element beamformer that is ideally set to the angle of arrival of the first multipath, the power of the first multipath is increased by $20 \log N$, which for larger $N$ might be sufficient for acceptable $S N R$.

As can be seen, a framework for description of IR UWB arrays has been already developed. However, there are some major issues with the definitions (9)-(11). These definitions depend strongly on the nature of used UWB signal $s(t)$. Studies performed in [64] show that the sidelobe level and well as the beam width in beam patterns $B P_{I}$ and $B P_{I I I}$ depend strongly on the pulse waveform. Furthermore, application of the radiation properties of individual antenna elements for case of nonomni-directional antennas is not very straightforward, especially as antenna impulse responses depend strongly on the antenna type and angle of arrival [31,32]. Lastly, the framework from $[64,66]$ assumes that the received signal can be assumed as a plane wave. Some simulations in [66] assume 8 -element array spaced by $3.63 \mathrm{~cm}$. The resulting the length such array is $25.41 \mathrm{~cm}$. With this array size, the plane wave condition for ranges below $2 \mathrm{~m}$ (typical for many UWB applications) the validity of approximation by plane will strongly depend on the length of pulse $s(t)$. As a result, one of the future challenges for the community is a development of a more general framework that will not be limited by issues mentioned above.

\subsubsection{Multiple Transmit Antennas}

As mentioned in the introduction to this section, UWB systems are EIRP constrained whereas most of the algorithms for MIMO/MISO systems have been developed for power constrained systems such as $802.11 \mathrm{n}$. Practically it means that whilst power constrained systems may benefit from increased gain as a result of beam forming, UWB systems have to keep the maximum gain so that the regulatory levels of EIRP spectral density are not violated. As a result, some of the algorithms known for power constrained systems must be adapted. The issue of EIRP constrained systems has been addressed by several authors e.g. in [65,67-69]. The main approaches studied by the literature are antenna selection and EIRP-/gain-constrained beamforming.

\section{Antenna Selection}

Systems with antenna selection transmit the information, or its part through one antenna in an array. A fundamental requirement for the success of this technique is the decorrelation of both antennas. This has been excessively studied and reported in reference [70], where it has been shown 
that the correlation between antennas decreases with distance, but surprisingly the decrease is more rapid for endfire arrays than for broadside arrays. For practical systems, the decorrelation also depends on the actual design of the antennas. Some practical designs of antennas for MIMO UWB systems are presented in [71,72].

References $[65,69]$ present this approach applied to WiMedia OFDM system applied to a subcarrier level. In other words, the channel transfer function between transmit antennas and the receiver are determined for every subcarrier and the antenna with the highest magnitude of channel transfer function is selected. This is an efficient way to reduce the impact of frequency fading as described in $[24-27,30]$. The selection requires the knowledge of channel state [65] but due to the reciprocity of the channel, this can be estimated by the multiple-antenna transceiver from the OFDM symbols sent by the single-antenna transceiver.

In this work, measurements from [27] have been used to analyse the potential BER improvement achievable by this approach in a Wimedia system. The BER rate is evaluated on the lowest physical layer with no channel coding. In other words, the mean BER is evaluated for 128 QPKS channels with 4.125 MHz bandwidth centred around $6.072 \mathrm{GHz}$ (centre frequency of WiMedia Band 6 [11]). One set of measurements from [27] is used. This set represents 1,600 channel transfer functions in a grid of $40 \times 40$ points spaced by $6 \mathrm{~mm}$. For the selected measurement set, the distance between the grid centre and the transmitter was $1.93 \mathrm{~m}$. The measurements were conducted in a laboratory environment and more details on the actual measurements can be found in reference [27]. The BER simulation results are presented in Figs. 6-8.

Figure 6 presents an example of BER curve improvement for two antennas spaced by $4.2 \mathrm{~cm}$ compared to the SISO case. Fig. 7 then presents the impact of distance on performance improvement. The channel measurements were used to simulate $1,000(=40 \times 25)$ 2-element broadside arrays with variable distance between $0.6 \mathrm{~cm}$ and $9 \mathrm{~cm}$. These 1,000 empirical channel were used to determine the mean $E_{b} / N_{0}$ gain for BER of $10^{-5}$ compared to the SISO case. The results confirm the conclusion of [70] as the gain increases with element distance until saturation at the level of $10 \mathrm{~dB}$ is reached for element spacing by approx. $70 \mathrm{~mm}$. Fig. 7 provides a useful insight what gain can be expected for a selected element spacing which in practical systems will be determined by the size of the device. This gain can then be enter the trade-off considerations between gain and the increase in system complexity.

Finally, Fig. 8 presents the impact of increased number of antennas on the performance. Again, the mean $E_{b} / N_{0}$ gain for $B E R$ of $10^{-5}$ compared to the SISO case is presented for various number of array elements. The measurements were used to model 40 cases for broadside antenna arrays spaced by $4.2 \mathrm{~cm}$. The number of elements in the arrays was varied from 1 (SISO case) to 6 . Similarly 
to the case when element spacing is varied the gain increases with the number of elements because a higher number of de-correlated channels is available. Saturation is reached for the level of approx. $12 \mathrm{~dB}$. On the other hand, the complexity of antenna selection increases with the number of antennas. Thus, Fig. 8 provides a quantified input for this trade-off decision.

References $[65,69]$ further note the fact that the subcarriers will no longer have continuous phase. This may cause issues in cases of a legacy WiMedia receivers unaware of the multiple transmit antenna. If it employs signal processing algorithms that assume continuous phase may actually degrade the performance. Thus, antenna selection with phase pre-coding is suggested in $[65,69]$. Further point risen by [65] is the load of power amplifiers which might not be equally loaded reducing the energy efficiency of the transmitter. Thus algorithm considering equal loading of the amplifiers can be employed as presented in [65].

As can be seen in Figs. 6-8, even relatively low complex antenna selection can significantly reduce the impact of frequency selective fading and significantly reduce the required $E_{b} / N_{0}$. As a result, significantly higher data-rates and/or longer range and/or lower complexity receiver can be achieved. The antenna selection is suitable even for non-WiMedia modulation schemes. The gain might be lower for wideband schemes such as MTFSK, SC FDE or IR UWB because these schemes are inherently less affected by frequency selective fading (see Section 5.2), but they can still profit from gain due to spatial diversity.

\section{Beamforming}

Whilst antenna selection offers a significant improvement of BER performance, further improvement can be achieved by the use of beamforming. This can be explained using Section 5.2.1. Even with antenna selection, the RF energy is radiated in all directions. Hence, the constructive and destructive multipaths remain present at the receiver. However, a beamformer can be used to direct energy in the constructive multipath components only. This will increase the SNR at the receiver even though the total radiated energy will actually be reduced because it will not be radiated omni-directionally. As shown in Section 5.4.1 the beamforming strategy depends strongly on actual modulation scheme and is fundamentally different for WiMedia OFDM and for Impulse Radio. This section focuses mainly on beamforming in Wimedia OFDM systems. The basic concept of beamforming for IR UWB using adaptive delay lines has already been introduced in Section 5.4.1. Further details on this topic can be found in [64] and references therein.

As mentioned in the introduction of this Section, standard beamforming algorithms such as Eigenbeamforming cannot be used in case of UWB because they have been developed for power constrained systems where EIRP in a certain direction can be increased. As shown in [73], the EIRP constrained beamforming can be solved as a convex optimisation problem, which is unfortunately 
computationally complex $[67,73]$. Henceforth, references $[67,73]$ suggest a computationally less demanding suboptimal use of Eigen-beamforming where the final result is scaled so that the EIRP limits are not violated.

Unfortunately according to [65], scaled Eigen-beamforming's BER performance is inferior antenna selection. On the other-hand, $[65,74]$ show that the lobes of antenna factor generated by scaled Eigen-beamforming have the same spatial distribution as the lobes of the optimal scheme, even though their level is lower. Therefore, $[65,74]$ suggest a new method that uses the result of scaled Eigen-beamforming and reduces the spatial peak to average ratio of the array factor to obtain a suboptimal method with BER performance closer to the optimal scheme than antenna selection or scaled-eigenbeamforming. Performance of this method is shown significantly superior to antenna selection.

To conclude, beamforming for WiMedia OFDM systems on the transmitter side is complex due to the EIRP limitations. The optimum solution to the problem is known, but it is computationally complex. Even though sub-optimal solutions have been proposed in $[67,68,73,74]$, future research into low complexity optimum EIRP constrained beamforming remains very attractive.

\subsubsection{Multiple Receive-Antennas}

The case of multiple receiver antennas is almost equivalent to the case multiple transmit antenna with the advantage that EIRP constrained are relaxed, which allows more flexibility for both: diversity combining and beamforming.

Developing the concept, diversity combining antenna selection can be used similarly to the transmit case and due to the reciprocity of the wireless channel. Further performance improvement can be achieved if the signal from receiver antennas is combined. There are several strategies for signal combining, e.g., equal gain combining, maximum ratio combining, which differ in performance and complexity [70]. To illustrate the advantage of combining over antenna selection, the measurements from [27] was used to model receiver array. The simulation presented in Fig. 7 has been repeated but the signals were combined in optimal manor. As a result, Fig. 9 presents the $E_{b} / N_{0}$ gain for $B E R$ of $10^{-5}$ compared to the SISO case for antenna selection and maximum ratio combining. It can be seen that the mean available gain for uncorrelated channel increases approximately by further $2 \mathrm{~dB}$. Not only can multiple antennas at the receiver be used for beam forming with no constraint on total gain, they can also suppress interferers by null steering. Therefore, optimum techniques well known for narrowband systems e.g. [62] can be used to obtain a better performance. However, a specific of UWB Wimedia systems is the computational complexity discussed in section 5.1. Therefore, investigations of sub-optimal low complexity RX beamformers have been conducted. For instance, [75] suggest a low complexity beamformer limiting the phase space available for signal combining to 
8 (3-bit). Furthermore, the same phase will be applied to all the subcarriers. Despite the low complexity, initial results in [75] suggest significant performance improvement.

\subsubsection{Conclusion}

This section has discussed the MISO and SIMO systems individually because due to the EIRP constraints, they are not equivalent. This difference and its impact on used algorithm is explained. The combination of both into a MIMO system is possible and is being investigated. In a sense it is a straightforward combination of SIMO and MISO cases and is therefore not discussed here.

Further interesting prospect and research avenue is the use of multiple antenna systems to increase the security of UWB wireless communication as presented in [63] for narrowband cases. The main idea is to use the unique spatially specific channel information available only to the two transceivers as a part of security key.

\subsection{Radio over Fibre}

One of the main drawbacks of the UWB technology is its limited range that is typically below $10 \mathrm{~m}$ within a single room $[5,6,11]$. As a result, it cannot compete with 802.11 standard in distributing wireless connectivity throughout a building. To overcome this issue, the Radio-over-Fibre technology can be used. Radio-over-Fibre is an important tool for future UWB systems as well for current research.

Currently, UWB Radio-over-Fibre (RoF) links can be used to replace cables in UWB wireless channel sounding. Such technology was used e.g. in [27]. Channel measurements for frequencies around and above $10 \mathrm{GHz}$ are extremely challenging as the loss introduced by RF cable increases with frequency. With RoF links, this challenge can be addressed since the advantage is that a wideband RoF link represents approximately a constant gain or attenuation over the entire frequency span for bandwidths of up to $20 \mathrm{GHz}$ enabling measurements impossible with standard coaxial cables [27].

For the future, the use of RoF as part of UWB communication systems is considered e.g. by the EU project Ultrawideband Radio over Optical Fiber [76]. This project studies future systems and discusses architectures that might provide the platform for the requirements of such systems. UWB RoF are considered e.g. as distribution network for wireless connectivity throughout buildings. The focus is mainly on the exploration and comparison of various topologies of the backbone Fibre network [77]. UWB RoF, however, can be employed in a wider spectrum of applications. Reference [76], for instance, mentions homeland security. Many pieces of infrastructures such as tunnels require constant supervision by CCTVs. Currently, there has to be a link between the supervisor's station and every single camera, which are often densely located. In the future, these cameras might be connected wirelessly to one UWB remote access point which will transfer the data to the 
supervisor's station via a single optical fibre. Such architecture would significantly increase the flexibility and reduce the complexity of such networks [76].

Even though UWB RoF links are already considered as part of future systems, there is still significant amount of research optimising the performance of individual links. A very good summary of the state-of-the-art research in this field is reference [78]. For instance, different modulation schemes (direct laser modulation or various external modulation) are compared in terms of performance (gain, noise figure, spurious free dynamic range, cost etc.) [78] and each of these approaches is being further improved e.g. [79-81].

In general, the RoF links are well understood and developed. However, one of the future challenges is to bring together the UWB and RoF links. Whilst RoF links already provide the bandwidth required for UWB devices [78], they have been developed using the standard approach of RF engineering with the aim to maximise gain and minimise noise figure. As pointed out in [82], the gain maximisation is not so important for UWB. In fact, when the network architectures proposed in [77] are considered, designs should aim for a gain of $0 \mathrm{~dB}$, whilst minimising the noise figure [82]. The choice of $0 \mathrm{~dB}$ is made because some of the architectures in [77] re-transmit the signal over two or more RoF links. With a positive gain, the RoF link could be overloaded or the EIRP spectral limits could be violated. With negative gain, the range of the signal and system performance would be compromised. Thus, new design rules for RoF links need to be further developed using the body of knowledge gather for conventional designs maximising gain $[78,79]$.

\subsection{Higher band UWB}

Following rather strict regulatory rulings in Europe and Asia, some researchers have started to look at the less-utilised parts of the spectrum for implementing UWB techniques without the harsh restrictions imposed on the $3.1-10.6 \mathrm{GHz}$ band. The spectrum around the unlicensed $60 \mathrm{GHz}$ band (e.g. $57-64 \mathrm{GHz}$ in United States) is currently one of the hot candidates for a revival of the UWB technology [83-85]. The choice of higher band has both advantages and disadvantages. In terms of advantages, there is no narrowband interference by primary users as the band and it allows higher density of frequency re-use which increases the total data throughput in an environment [84]. The main disadvantage is the increased path loss that needs to be compensated by beamforming. These issues will be discussed below. Despite these issues, recent progress has already allowed fully integrated CMOS solutions [85] and several standards, introduced below, have been approved.

As mentioned above, the biggest disadvantage of is the path loss. Compared to $6 \mathrm{GHz}$, the $60 \mathrm{GHz}$ band automatically introduces a $20 \mathrm{~dB}$ penalty in path loss which compromises the range and/or the achievable data-rate. On the other hand, the path loss penalty can be compensated by 
beamforming. The actual dimensions of the array will be small due to the small free space wavelength of a $60 \mathrm{GHz}$ system $(5 \mathrm{~mm}$ ) and the known relationship between the effective area and the gain of an antenna [86]:

$G=\frac{4 \pi}{\lambda_{0}^{2}} A_{e f f}$

where $G$ is the antenna gain, $A_{e f f}$ is the effective area of the antenna, $\lambda_{0}$ is the free space wavelength

For instance an array with an effective area of $2 \mathrm{~cm}^{2}$ (square $1.41 \times 1.41 \mathrm{~cm}$ ) can provide a gain of 20 $\mathrm{dB}$. For a gain of $30 \mathrm{~dB}$, the effective area is $20 \mathrm{~cm}^{2}$ (square $4.46 \times 4.46 \mathrm{~cm}$ ). From the design perspective, such dimensions do not impose any significant limitations. Furthermore, the beamforming at $60 \mathrm{GHz}$ is less complex tahn UWB beamforming because whilst the absolute bandwidth remains large, the relative bandwidth is approx. only $10 \%$. Therefore, the radiation pattern does not change significantly over the bandwidth and standard beamforming techniques can be used similarly to the case of one WiMedia band (Section 5.4.2).

Not only does beamforming compensate the path loss, it also positively impacts the performance and achievable data-rates. Firstly, the energy is only radiated in a narrow angle which reduces the number of significant multipaths at the receiver. Secondly, it reduces the mutual interference between neighbouring systems [84]. This is in more detail shown reference [84] where the frequency re-use of $60 \mathrm{GHz}$ band in an $9 \times 9 \mathrm{~m}$ office environment is studied. According to [84], if only one antenna element with $3 \mathrm{dBi}$ gain is used, only 2 active links with aggregated data-rate of 2 Gbps can operate in the studied office. With a 16-element array of the same antennas, 7 links can be used increasing the aggregated data-rate to $15 \mathrm{Gbps}$ [84].

Unfortunately, there are also two issues associated with beamforming. Firstly, it is the system complexity. Importance of the complexity and its impact on chip cost will be reduced as the technology will mature. Secondly, it is the risk of cluttering the main beam by pedestrians. This is a serious issue even for UWB systems operating in the $3.1-10.6 \mathrm{GHz}$ [87] and it can be anticipated that it will become more severe for the $60 \mathrm{GHz}$. As a result, systems have to implement sufficient measures to combat this issue.

Despite the issues, the $60 \mathrm{GHz}$ spectrum has attracted a significant amount of attention of academia and industry. As a result, some standards have been introduced recently. Unfortunately, it appears that the history of the battle of UWB standards is going to be repeated because up to date, the authors are aware of 3 standards: IEEE802.15.3c-2009 has been amended in 2009 to include section 
12 specifying the PHY of $60 \mathrm{GHz}$ band [88]; ECMA-387 has first been published in 2009 [89], currently there is version 2 from 2010; lastly industrial consortium Wireless $\mathrm{HD}^{\mathrm{TM}}$ (including e.g. Intel, Toshiba, Sony, Samsung) has published a standard for wireless streaming of high definition video in 2010 [90]. The main similarities and differences of the standard are briefly summarised here. All standards agree on division of the $60 \mathrm{GHz}$ band into 4 subbands and suggest the same spectral mask. However, they differ in the modulation scheme. Two competing schemes are proposed Single Carrier Block Transmission (SCBT), see section 5.2 for details, and OFDM.

IEEE802.15.3c-2009 suggests the use of SCM for low data-rate applications whilst high and medium data rates will use OFDM [89]. The highest achievable data-rate is 5.005 Gbps. The ECMA-387 is the most ambitious standard in terms of data-rate, SCBT is the main for the entire set of available datarates $(0.8-6.350 \mathrm{Gbps})$ as it has more relaxed requirements on amplifiers [83] (lower peak to average ratio discussed in section 5.2) and lower requirements on resolution of ADCs and DACs [83]. OFDM is also supported by ECMA-387 but it is not the main modulation scheme. Furthermore, bonding of channels is possible for the SCBT transmission $[83,89]$ allowing potentially data-rates up to $25.402 \mathrm{Gbps}$ [89]. WirelessHD ${ }^{\mathrm{TM}}$ on the other hand proposes the sole use of OFDM. In the high speed mode, the symbol duration will be 225.96 ns with FFT length of 512 [90]. The highest achievable data-rate is $7.138 \mathrm{Gbps}$ [90].

To conclude, this section presents the attempts to use the experience of UWB in a higher frequency band. The advantages (no narrowband interference, denser frequency re-use and higher aggregated data-rate) and the issues (complex beam forming, cluttering of the beam) are discussed. Finally, a brief overview over the recent standardisation efforts is provided.

\section{Discussion}

As stated in the first section of this paper, the main objective of this review is to briefly summarise the progress UWB has undergone in the past 20 years and to identify the main challenges that have to be addressed so that UWB can fully live up to its potential. Despite recent drawbacks caused by the market meltdown and delays in delivering products to marketplace, UWB is still considered as a technology worthy of interest because it is one of few technologies that have the potential to resolve the spectrum gridlock and to deliver "gigabit wireless".

The main issues of current UWB wireless systems that have been identified are: system complexity/cost; range; reliability/BER performance; data-rate; and the risks associated with strong narrowband interference. These issues have been addressed by recent research efforts identified in section 5 . The key findings are now summarised.

The inherent complexity of wideband OFDM is identified as the reason for system complexity and cost. This is being addressed by research into low complexity chip design, maturing of the 
technology, and alternative non-OFDM modulations such as single-carrier frequency domain equalization (SC FDE), multi-tone frequency shift keying (MTFSK) and impulse radio (IR) for low datarate applications. IR systems also suffer from complexity issue if coherent rake receivers are used, hence low complexity non-coherent receiver architectures are studied.

The range of systems is limited mainly by the link budget which is given by the combination regulations of EIRP spectral density, the noise floor, and the frequency selective fading. The main solution to this range issue may be the use of Radio-over-Fibre technologies and multiple antenna systems.

It is shown that the BER performance and data-rate are closely related. OFDM systems are suboptimal as these systems do not exploit the frequency diversity of UWB wireless channels directly, but by the means of complex channel coding which compromises the data-rate. It is shown that the frequency diversity is exploited through wideband modulation schemes such as SC FDE or MTFSK. As a result, higher net data-rate can be achieved as weaker channel coding is sufficient. This is underlined by the fact that SC transmission is already in use by some commercial products and is considered in some $60 \mathrm{GHz}$ standards. $60 \mathrm{GHz}$ wireless is also another approach to increase the datarate and it is shown that as it provides the possibility of denser frequency re-use giving a higher aggregated data-rate. Finally, it is shown that even low complexity multiple antenna techniques such as antenna selection have the potential for significant improvement of the gross BER performance of WiMedia OFDM systems (gain of $10 \mathrm{~dB}$ ) enabling higher data-rates for the same channel conditions. Weak narrowband interference is shown not to influence UWB systems as it manifests itself in a manner similar to frequency selective fading. Issues are associated with overloading of front ends by strong interferers in proximity. This issue has two levels - the detection of an interferer and its suppression. It is shown that the WiMedia standard posses the means to detect such case and avoid or suppress the affected sub-band. The research of detect and avoid algorithms (DAA) is also fuelled by regulations that restrict some bands only for users with DAA. The WiMedia's DAA algorithm is described. In terms of suppression the use of band-notch antennas and band-notch filters are the most popular alternatives. Switchable band-notch antennas/filters are proposed to be used jointly with detect algorithms. Another alternatives discussed is null steering in multiple antenna systems and the use of $60 \mathrm{GHz}$ systems which currently avoid narrowband interferers.

\section{Conclusion}

In the past 20 years, UWB wireless systems have evolved from theoretical considerations into real products competing in the commercial arena. This paper comprehensively reviews these twenty years and discusses UWB's future. Indisputable achievements of UWB are acknowledged, but it is shown that the performance of current systems is yet to live up to the potential of gigabit-wireless 
expected in the beginnings of the technology. It is shown, however, that there remains significant academic and commercial interest in the technology. The gap between the achieved and potential performance is well understood and addressed by leading researchers around the globe.

A specific feature of this review is the cross-layer approach. The latest findings from different system layers, e.g., wave propagation, are linked to the system performance or complexity. The authors believe that these links provide more understanding for the opportunities and challenges of UWB technology that is, in many aspects, fundamentally different to classical narrowband communications.

The main contribution of this review is that it identifies what the authors consider to be the most serious challenges the UWB technology currently faces. The underlying reasons are explained based on the latest scientific findings or the limits of the state-of-the-art technology and the main research avenues addressing the challenges are introduced to show that the gap is being closed and UWB can still live up to its initial potential. 


\section{References:}

[1] Susskind, C.; , "Heinrich Hertz: a short life," Microwave Theory and Techniques, IEEE Transactions on , vol.36, no.5, pp.802-805, May 1988

[2] Falciasecca, G.; Valotti, B.; , "Guglielmo Marconi: The pioneer of wireless communications," Microwave Conference, 2009. EuMC 2009. European , vol., no., pp.544-546, Sept. 29 2009-Oct. 12009

[3] Goldsmith, A.; Jafar, S.A.; Maric, I.; Srinivasa, S.; , "Breaking Spectrum Gridlock With Cognitive Radios: An Information Theoretic Perspective," Proceedings of the IEEE , vol.97, no.5, pp.894914, May 2009

[4] Čabrić, D.; Mishra, S. M.; Willkomm, D.; Brodersen, R.; Wolisz, A.; , "A Cognitive radio approach for usage of virtual unlicensed spectrum," In Proc. of 14th IST Mobile Wireless Communications Summit, 2005

[5] Roy, S.; Foerster, J.R.; Somayazulu, V.S.; Leeper, D.G.; , "Ultrawideband radio design: the promise of high-speed, short-range wireless connectivity," Proceedings of the IEEE , vol.92, no.2, pp. 295311, Feb 2004

[6] B. Allen et. al. Ultra Wideband Antennas and Propagation for Communications, Radar and Imaging. John Wiley \& Sons, 2006

[7] Fullerton, L.; , "UWB waveforms and coding for communications and radar," Telesystems Conference, 1991. Proceedings. Vol.1., NTC '91., National , vol., no., pp.139-141, 26-27 Mar 1991

[8] Win, M.Z.; Scholtz, R.A.; , "Impulse radio: how it works," Communications Letters, IEEE , vol.2, no.2, pp.36-38, Feb 1998

[9] Multiband OFDM Alliance; "Ultrawideband: High-speed, short-range technology with farreaching effects," MBOA White Paper, September 1, 2004

[10] Halper, M.; "Future Focus, " TIME, Dec. 6, 2004

[11] G. Heidari, WiMedia UWB - technology choice for wireless USB and Bluetooth. John Wiley \& Sons, 2008.

[12] G. Fleishman; "UWB group shutters, sends tech to Bluetooth, USB groups," Ars Technica, March 2009

[13] R. Merrit; "UWB start-up Radiospire shuts down, " EE Times, April 2009

[14] Ofcom; "Decision to make the Wireless Telegraphy (Ultra-Wideband Equipment) (Exemption) Regulations 2009," Ofcom, 2009

[15] Berens, F.; , "The European flexible DAA approach towards an open UWB regulation," UltraWideband, 2008. ICUWB 2008. IEEE International Conference on , vol.3, no., pp.71-75, 10-12 Sept. 2008

[16] US FCC; Ruling Part 15, US FCC, February 2002

[17] Liu, L.; Dong, X.; Tian, Z.; Schwartz, A.L.; , "Link Budget Analysis and Throughput Measurement for Multi-Antennas WiMedia UWB Systems," Vehicular Technology Conference Fall (VTC 2009Fall), 2009 IEEE 70th , vol., no., pp.1-5, 20-23 Sept. 2009

[18] Johnson, S. G.; Frigo, M.; , "A Modified Split-Radix FFT With Fewer Arithmetic Operations," Signal Processing, IEEE Transactions on , vol.55, no.1, pp.111-119, Jan. 2007

[19] Vouzis, P.; Arnold, M.G.; Paliouras, V.; , "Using CLNS for FFTs in OFDM demodulation of UWB receivers," Circuits and Systems, 2005. ISCAS 2005. IEEE International Symposium on , vol., no., pp. 3954- 3957 Vol. 4, 23-26 May 2005 
[20] Cho, S.I.; Kang, K.M.; , "A Low-Complexity 128-Point Mixed-Radix FFT Processor for MB-OFDM UWB Systems," ETRI Journal, pp. 1-10, Vol. 32, Feb 2010

[21] Minhyeok Shin; Hanho Lee; , "A high-speed four-parallel radix-24 FFT/IFFT processor for UWB applications," Circuits and Systems, 2008. ISCAS 2008. IEEE International Symposium on , vol., no., pp.960-963, 18-21 May 2008

[22] Sherratt, R.S.; Cadenas, O.; Goswami, N.; , "A low clock frequency FFT core implementation for multiband full-rate ultra-wideband (UWB) receivers," Consumer Electronics, IEEE Transactions on , vol.51, no.3, pp. 798- 802, Aug. 2005

[23] Sherratt, R.S.; Makino, S.; , "Numerical precision requirements on the multiband ultra-wideband system for practical consumer electronic devices," Consumer Electronics, IEEE Transactions on , vol.51, no.2, pp. 386- 392, May 2005

[24] Romme, J.; Kull, B.; , "On the relation between bandwidth and robustness of indoor UWB communication," Ultra Wideband Systems and Technologies, 2003 IEEE Conference on , vol., no., pp. 255- 259, 16-19 Nov. 2003

[25] Malik, W.Q.; Allen, B.; Edwards, D.J.; , "Fade depth scaling with channel bandwidth," Electronics Letters, vol.43, no.24, pp.1371-1372, Nov. 222007

[26] Malik, W.Q.; Allen, B.; Edwards, D.J.; , "Bandwidth-dependent modelling of smallscale fade depth in wireless channels," Microwaves, Antennas \& Propagation, IET , vol.2, no.6, pp.519-528, Sept. 2008

[27] Sipal, V.; Allen, B.; Edwards, D.; "Exploration and analysis of fade depth scaling," Antennas and Propagation Conference (LAPC), 2010 Loughborough , vol., no., pp.125-128, 8-9 Nov. 2010

[28] Wang, Y.; Dong, X.; Wittke, P.H.; Shaomin Mo; , "Cyclic prefixed single carrier transmission in ultra-wideband communications," Wireless Communications, IEEE Transactions on , vol.5, no.8, pp.2017-2021, Aug. 2006

[29] Sipal, V.; Allen, B.; Edwards, D.; " Empirical Study of Multi-tone Frequency Shift Keying for Ultrawideband Wireless Communications," Communications, IET, vol. 5, no., pp., 2011

[30] Sipal, V.; Allen, B.; Edwards, D.; " Frequency Selective Fading of Ultrawideband Wireless Channels in Confined Environments," Microwaves Antennas \& Propagation, IET, 2011 (in print)

[31] Sipal, V.; Allen, B.; Edwards, D.; , "Analysis and mitigation of antenna effects on wideband wireless channel," Electronics Letters, vol.46, no.16, pp.1159-1160, August 52010

[32] Sipal, V.; Allen, B.; Edwards, D.; , "Effects of antenna impulse response on wideband wireless channel," Antennas and Propagation Conference (LAPC), 2010 Loughborough , vol., no., pp.129132, 8-9 Nov. 2010

[33] Sipal, V.; Allen, B.; Edwards, D.; , "Exploration of Nakagami fading in ultra-wideband wireless channels," Electronics Letters, vol.47, no.8, pp.520 -521, April 142011

[34] Walzman, T.; Schwartz, M.; , "Automatic equalization using the discrete frequency domain," Information Theory, IEEE Transactions on , vol.19, no.1, pp. 59- 68, Jan 1973

[35] Falconer, D.; Ariyavisitakul, S.L.; Benyamin-Seeyar, A.; Eidson, B.; , "Frequency domain equalization for single-carrier broadband wireless systems," Communications Magazine, IEEE , vol.40, no.4, pp.58-66, Apr 2002

[36] Sari, H.; Karam, G.; Jeanclaud, I.; , "Frequency-domain equalization of mobile radio and terrestrial broadcast channels," Global Telecommunications Conference, 1994. GLOBECOM '94. Communications: The Global Bridge., IEEE , vol., no., pp.1-5 vol.1, 28 Nov- 2 Dec 1994 
[37] Sheng Li; de Lamare, R.C.; , "Adaptive Detector for SC-FDE in Multiuser DS-UWB Systems Based on Structured Channel Estimation with Conjugate Gradient Algorithm," Vehicular Technology Conference (VTC 2010-Spring), 2010 IEEE 71st, vol., no., pp.1-5, 16-19 May 2010

[38] Wang, Y.; Dong, X.; , "A time-division multiple-access SC-FDE system with IBI suppression for UWB communications," Selected Areas in Communications, IEEE Journal on , vol.24, no.4, pp. 920- 926, April 2006

[39] Wang, D.; Jiang, L.G.; He, C.; , "Noise variance estimation and optimal sequences for channel estimation in SC-FDE UWB systems," Electronics Letters, vol.43, no.11, pp.621-623, May 24 2007

[40] Wang, Y.; Dong, X.; , "Frequency-Domain Channel Estimation for SC-FDE in UWB Communications," Communications, IEEE Transactions on , vol.54, no.12, pp.2155-2163, Dec. 2006

[41] Wang, Y.; Dong, X.; , "Comparison of Frequency Offset and Timing Offset Effects on the Performance of SC-FDE and OFDM Over UWB Channels," Vehicular Technology, IEEE Transactions on, vol.58, no.1, pp.242-250, Jan. 2009

[42] Mlinarsky, F.; Ziegler, J.; “UWB Test Report,” Tech. Report, Octoscope, 13 Dec. 2007

[43] Luo, C.; Medard, M.; Zheng, L.; , "On approaching wideband capacity using multitone FSK,"Selected Areas in Communications, IEEE Journal on , vol.23, no.9, pp. 1830-1838, Sept. 2005

[44] Luo, C.; "Communication for Wideband Fading Channels: on Theory and Practice," PhD. Thesis, MIT, 2006

[45] Di Benedetto, M.-G. et. al. UWB Communications Systems Comprehensive overview. Hindawi Publishing Corporation, New York, 2006

[46] He, N.; Tepedelenlioglu, C.; , "Performance analysis of non-coherent UWB receivers at different synchronization levels," Wireless Communications, IEEE Transactions on , vol.5, no.6, pp.12661273, June 2006

[47] Lovelace, W.M.; Townsend, J.K.; , "The effects of timing jitter and tracking on the performance of impulse radio," Selected Areas in Communications, IEEE Journal on , vol.20, no.9, pp. 16461651, Dec 2002

[48] Lottici, V.; D'Andrea, A.; Mengali, U.; , "Channel estimation for ultra-wideband communications," Selected Areas in Communications, IEEE Journal on , vol.20, no.9, pp. 16381645, Dec 2002

[49] Sahin, M.E.; Guvenc, I.; Arslan, H.; , "Optimization of energy detector receivers for UWB systems," Vehicular Technology Conference, 2005. VTC 2005-Spring. 2005 IEEE 61st, vol.2, no., pp. 1386- 1390 Vol. 2, 30 May-1 June 2005

[50] Zhang, R.; Dong, X.; , "Synchronization and Integration Region Optimization for UWB Signals with Non-coherent Detection and Auto-correlation Detection," Communications, IEEE Transactions on, vol.56, no.5, pp.790-798, May 2008

[51] Parihar, A.; Lampe, L.; Schober, R.; Leung, C.; , "Equalization for DS-UWB Systems-Part I: BPSK Modulation," Communications, IEEE Transactions on , vol.55, no.6, pp.1164-1173, June 2007

[52] Dai, Y.; Yao, J.; , "Optical Generation of Binary Phase-Coded Direct-Sequence UWB Signals Using a Multichannel Chirped Fiber Bragg Grating," Lightwave Technology, Journal of , vol.26, no.15, pp.2513-2520, Aug.1, 2008 
[53] Perez-Duenas, J.; Wanguemert-Perez, J.G.; Molina-Fernandez, I.; , "Novel modulation scheme and six-port based RAKE receiver for DS-UWB," Wireless Communications, IEEE Transactions on , vol.8, no.7, pp.3628-3633, July 2009

[54] Wang, C.; Ma, M.; Ying, R.; Yang, Y.; , "Narrowband Interference Mitigation in DS-UWB Systems," Signal Processing Letters, IEEE , vol.17, no.5, pp.429-432, May 2010

[55] Wen Tao Li; Xiao Wei Shi; Yong Qiang Hei; , "Novel Planar UWB Monopole Antenna With Triple Band-Notched Characteristics," Antennas and Wireless Propagation Letters, IEEE , vol.8, no., pp.1094-1098, 2009

[56] Weng, Y.F.; Cheung, S.W.; Yuk, T.I.; , "Triple band-notched UWB antenna using meandered ground stubs," Antennas and Propagation Conference (LAPC), 2010 Loughborough , vol., no., pp.341-344, 8-9 Nov. 2010

[57] Athukorala, L.; Rabbi, K.; Panagamuwa, C.; Vardaxoglou, J.C.; Philippakis, M.; Budimir, D.; , "Optically reconfigurable microstrip UWB bandpass filters," Antennas and Propagation Conference (LAPC), 2010 Loughborough, vol., no., pp.617-620, 8-9 Nov. 2010

[58] Mishra, S.M.; Brodersen, R.W.; Brink, S.T.; Mahadevappa, R.; , "Detect and avoid: an ultrawideband/WiMAX coexistence mechanism [Topics in Radio Communications]," Communications Magazine, IEEE , vol.45, no.6, pp.68-75, June 2007

[59] Yang, R.; Zhou, Z.; Zhang, L.; Yu, C.; , "Detection and avoidance scheme for direct-sequence ultra-wideband system: a step towards cognitive radio," Communications, IET , vol.2, no.8, pp.1043-1050, September 2008

[60] Mishra, S.M.; ten Brink, S.; Mahadevappa, R.; Brodersen, R.W.; , "Cognitive Technology for Ultra-Wideband/WiMax Coexistence," New Frontiers in Dynamic Spectrum Access Networks, 2007. DySPAN 2007. 2nd IEEE International Symposium on , vol., no., pp.179-186, 17-20 April 2007

[61] Facchini, F.; Giuliano, R.; Mazzenga, F.; , "Ultra-wideband detect and avoid procedure for WiMAX victims," Communications, IET, vol.3, no.2, pp.268-278, February 2009

[62] Jensen, M.A.; Wallace, J.W.; , "A review of antennas and propagation for MIMO wireless communications," Antennas and Propagation, IEEE Transactions on , vol.52, no.11, pp. 28102824, Nov. 2004

[63] Chan Chen; Jensen, M.A.; , "Secret Key Establishment Using Temporally and Spatially Correlated Wireless Channel Coefficients," Mobile Computing, IEEE Transactions on , vol.10, no.2, pp.205215, Feb. 2011

[64] Kaiser, T.; Feng Zheng; Dimitrov, E.; "An Overview of Ultra-Wide-Band Systems With MIMO," Proceedings of the IEEE, vol.97, no.2, pp.285-312, Feb. 2009

[65] Vithanage, C.; Sandell, M.; Coon, J.; Wang, Y.; , "Precoding in OFDM-based multi-antenna ultrawideband systems," Communications Magazine, IEEE , vol.47, no.1, pp.41-47, January 2009

[66] Ries, S.; Kaiser, T.; "Ultra wideband impulse beamforming: It is a different world," Signal Processing , vol.86,issue 9, pp.2198-2207, Feb. 2006

[66] Kuzminsky, A.M.; "Downlink beamforming subject to the equivalent isotropic radiated power constraint in WLAN OFDM systems," Signal Processing , vol.87,issue 5, pp. 991-1002, May 2007

[67] Vithanage, C.M.; Coon, J.P.; Parker, S.C.J.; , "On capacity-optimal precoding for multiple antenna systems subject to EIRP restrictions," Wireless Communications, IEEE Transactions on , vol.7, no.12, pp.5182-5187, December 2008 
[68] Vithanage, C.M.; Parker, S.C.J.; Sandell, M.; , "Antenna Selection with Phase Precoding for High Performance UWB Communication with Legacy WiMedia Multi-Band OFDM

Devices," Communications, 2008. ICC '08. IEEE International Conference on , vol., no., pp.39383942, 19-23 May 2008

[70] Malik, W.Q.; , "Spatial correlation in ultrawideband channels," Wireless Communications, IEEE Transactions on , vol.7, no.2, pp.604-610, February 2008

[71] Hong, S.; Chung, K.; Lee, J.; Jung, S.; Lee, S.S.; Choi, J.; "Design of a diversity antenna with stubs for UWB applications," Microwave and Optical Technology Letters, vol. 50, no. 5, pp.1352-1356, May 2008

[72] Zhou, F.; Qian, Z.; Liu, T.; Han, J.; Peng, C.; , "Design of diversity antenna for ultra-wideband applications," UItra-Wideband (ICUWB), 2010 IEEE International Conference on , vol.1, no., pp.14, 20-23 Sept. 2010

[73] Zetterberg, P.; Bengtsson, M.; McNamara, D.; Karlsson, P.; Beach, M.A.; , "Performance of multiple-receive multiple-transmit beamforming in WLAN-type systems under power or EIRP constraints with delayed channel estimates, "Vehicular Technology Conference, 2002. VTC Spring 2002. IEEE 55th , vol.4, no., pp. 1906- 1910 vol.4, 2002

[74] Vithanage, C.M.; Wang, Y.; Coon, J.P.; "Spatial PAPR Reduction Based Beamforming Scheme for EIRP Constrained Systems", Global Telecommunications Conference, 2008. IEEE GLOBECOM 2008. IEEE, On page(s): 1 - 5, Volume: Issue: , Nov. 30 2008-Dec. 42008

[75] Malik, W.Q.; Allen, B.; Edwards, D.J.; , "A Simple Adaptive Beamformer for Ultrawideband Wireless Systems," Ultra-Wideband, The 2006 IEEE 2006 International Conference on , vol., no., pp.453-457, 24-27 Sept. 2006

[76] R.Kraemer, M. Katz, Short Range wireless Communication Emerging Technologies and Applications, Wiley 2009, ch. 15

[77] "UROOF system scenarios: Topology and architecture", UROOF deliverable 2.2, http://www.fp7daphne.eu

[78] I. Cox, C.H., E. Ackerman, G. Betts, and J. Prince, "Limits on the performance of rf-over-fiber links and their impact on device design," Microwave Theory and Techniques, IEEE Trans., vol. 54, no. 2, pp. 906 - 920, Feb. 2006.

[79] H.V. Roussell, M.D. Regan; J.L. Prince, C.H. Cox.; J.X. et. al., "Gain, Noise Figure and BandwidthLimited Dynamic Range of a Low-Biased External Modulation Link," Microwave Phot., 2007 IEEE Inter. Meeting on , vol., no., pp.84-87, 3-5 Oct. 2007

[80] G. Betts, X. Xie, I. Shubin, W. Chang, and P. Yu, "Gain limit in analog links using electroabsorption modulators," Photonics Technology Letters, IEEE, vol. 18, no. 19, pp. 2065 2067, Oct.1, 2006.

[81] J.X. Chen; Y. Wu; W.X. Chen; I. Shubin; A. Clawson; W.S.C. Chang; P.K.L. Yu; , "High-power intrastep quantum well electroabsorption modulator using single-sided large optical cavity waveguide," Phot. Techn. Lett., IEEE , vol.16, no.2, pp.440-442, Feb. 2004

[82] Sipal, V.; Allen, B.; Edwards, D.; "Optimisation Low Power Radio-over-Fibre Links using EAM for UWB Systems," International Conference on UWB ICUWB 2011, Bologna, 14-16 Sep. 2011

[83] Seyedi, A.; "On the physical layer performance of Ecma-387: A standard for $60 \mathrm{GHz}$ WPANs," Ultra-Wideband, 2009. ICUWB 2009. IEEE International Conference on , vol., no., pp.28-32, 9-11 Sept. 2009 
[84] Minyoung Park; Gopalakrishnan, P.; , "Analysis on spatial reuse, interference, and MAC layer interference mitigation schemes in $60 \mathrm{GHz}$ wireless networks," Ultra-Wideband, 2009. ICUWB 2009. IEEE International Conference on , vol., no., pp.1-5, 9-11 Sept. 2009

[85]_Laskar, J.; Pinel, S.; Dawn, D.; Sarkar, S.; Sen, P.; Perunama, B.; Yeh, D.; Barale, F.; , "60GHz entertainment connectivity solution," Ultra-Wideband, 2009. ICUWB 2009. IEEE International Conference on , vol., no., pp.17-21, 9-11 Sept. 2009

[86] Balanis, C.A.; Antenna Theory, Analysis and Design. John Wiley \& Sons, 2nd ed., 1997.

[87] Ali, A.J.; Scanlon, W.G.; Cotton, S.L.; , "Pedestrian effects in indoor UWB off-body communication channels," Antennas and Propagation Conference (LAPC), 2010 Loughborough , vol., no., pp.57-60, 8-9 Nov. 2010

[88] IEEE802.15.3c-2009 standard, http://ieeexplore.ieee.org/stamp/stamp.jsp?tp=\&arnumber=5284444\&tag=1, accessed July 2011

[89] ECMA-387 v.2 standard, http://www.ecma-international.org/publications/files/ECMAST/ECMA-387.pdf, accessed July 2011

[90] WirelessHD ${ }^{\mathrm{TM}}$ Specification, http://www.wirelesshd.org/pdfs/WirelessHD-SpecificationOverview-v1.1May2010.pdf, accessed July 2011 
List of Figures:
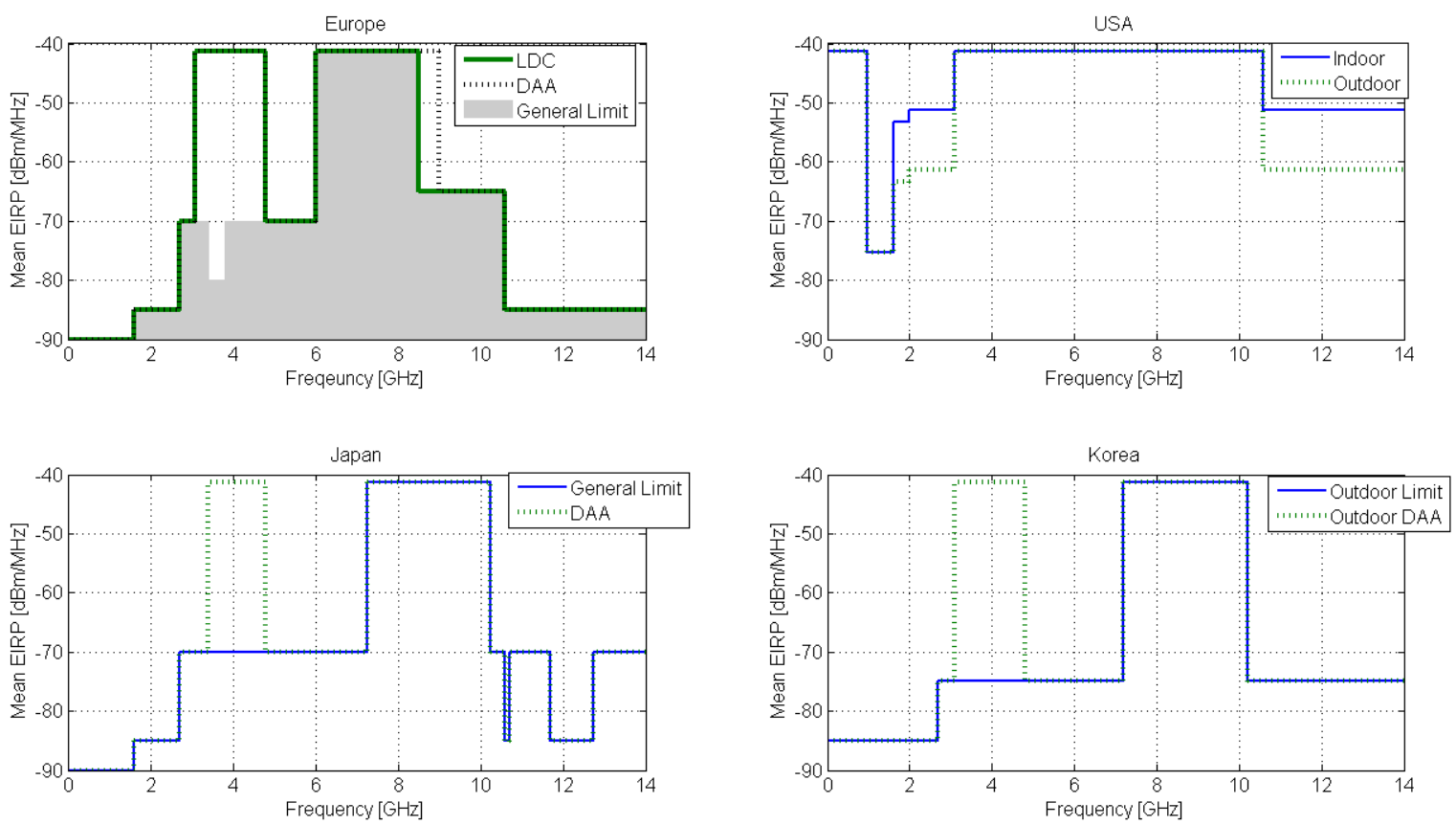

Fig. 1 UWB spectrum emission limits

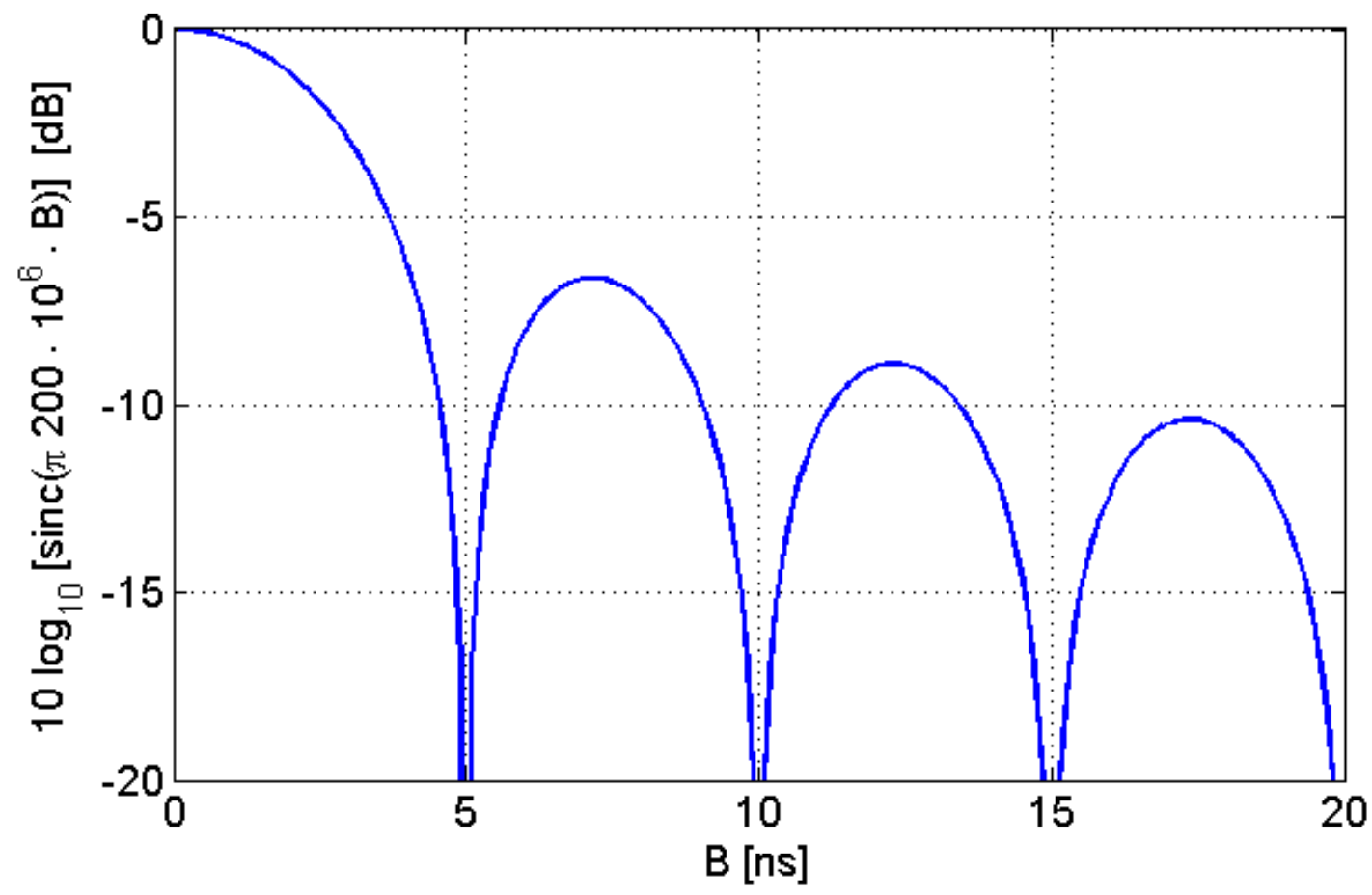

Fig. 2 The magnitude of the sinc-term from Eq. (5) for bandwidth of $200 \mathrm{MHz}$. 


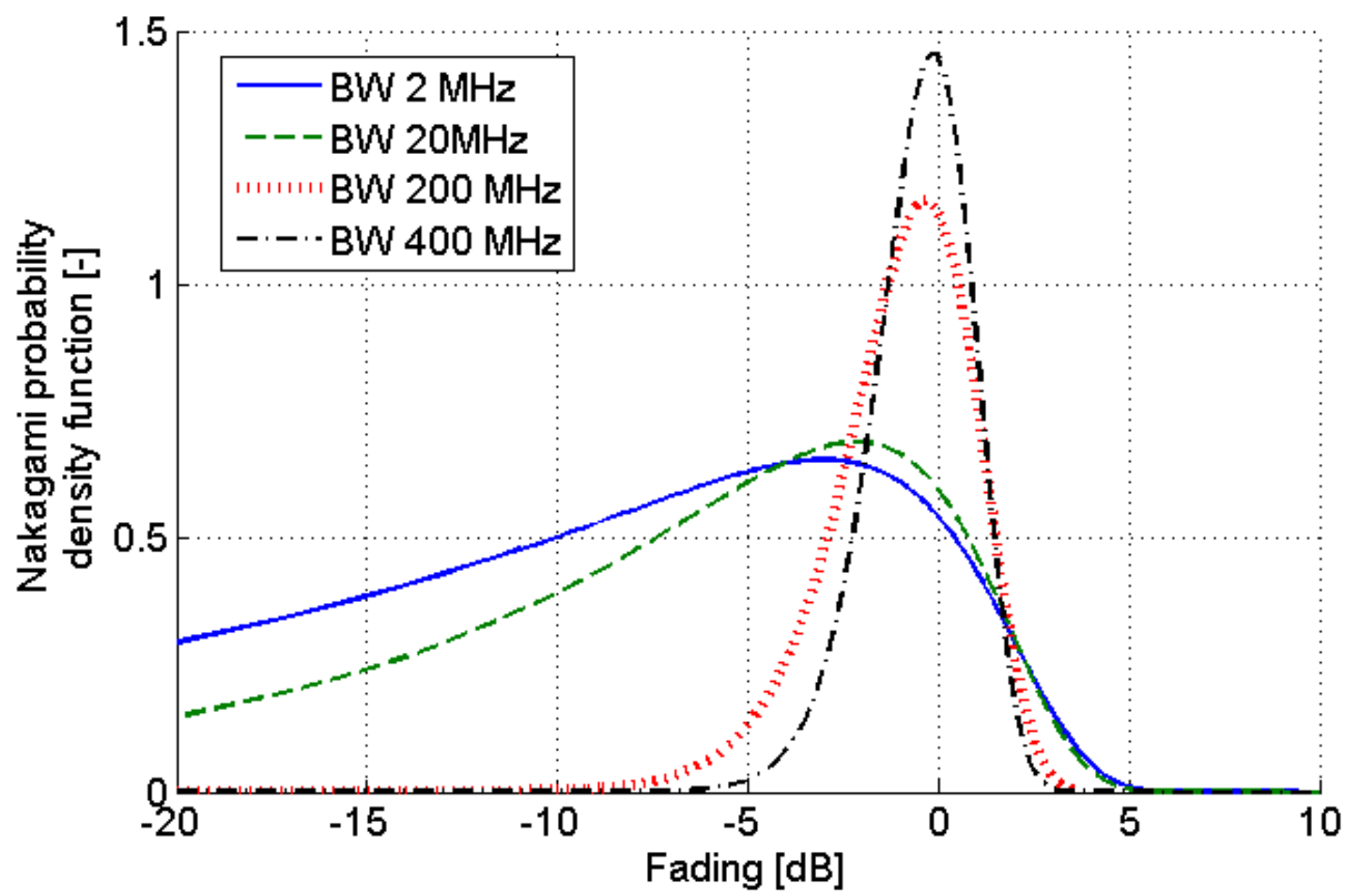

Fig. 3 The change of the shape of Nakagami probability distribution function for fading with the increase of signal bandwidth illustrates the increase of robustness of the signal to the frequency selective fading. Figure inspired by [32]

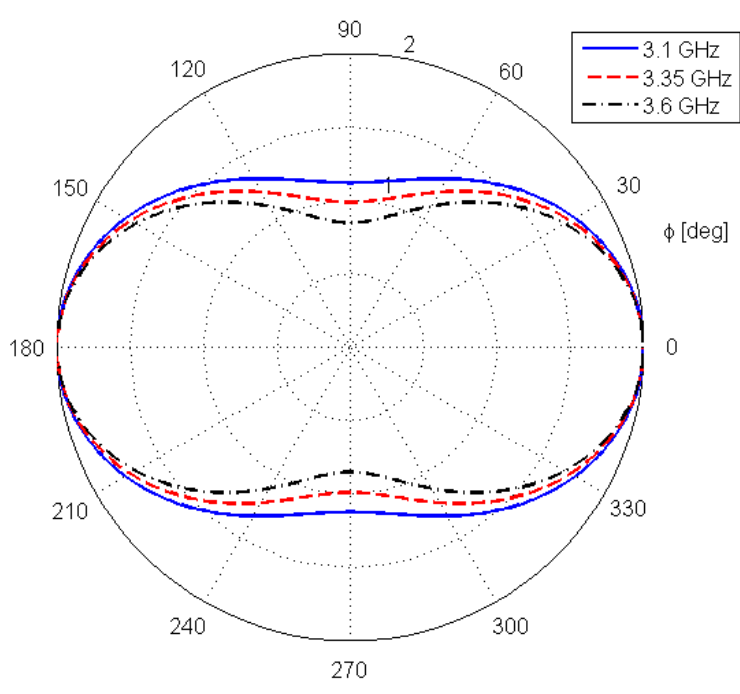

a)

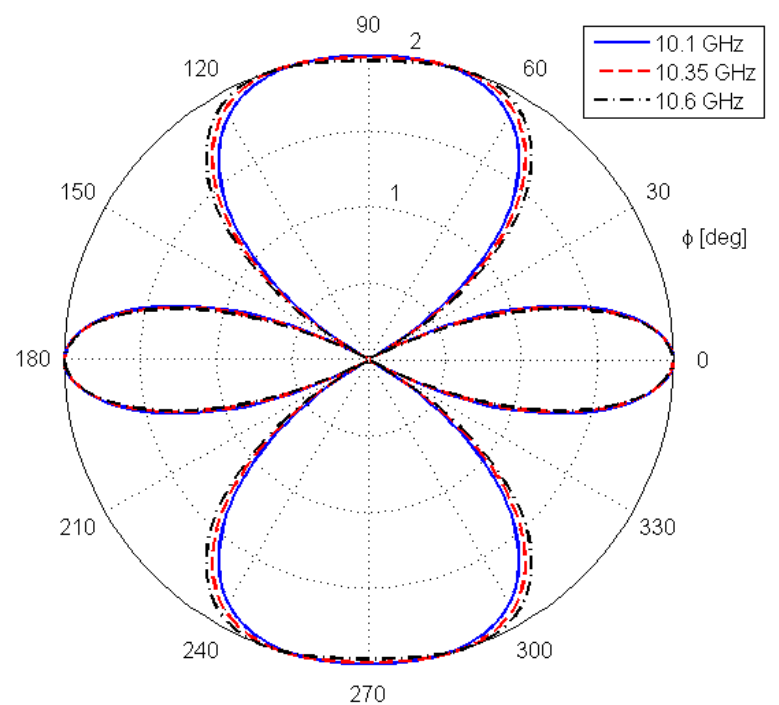

b)

Fig. 4 Array factor for frequencies at (a) the lower and (b) the upper end of FCC UWB spectrum.

Element spacing is $3 \mathrm{~cm}$, number of elements is 2 . 


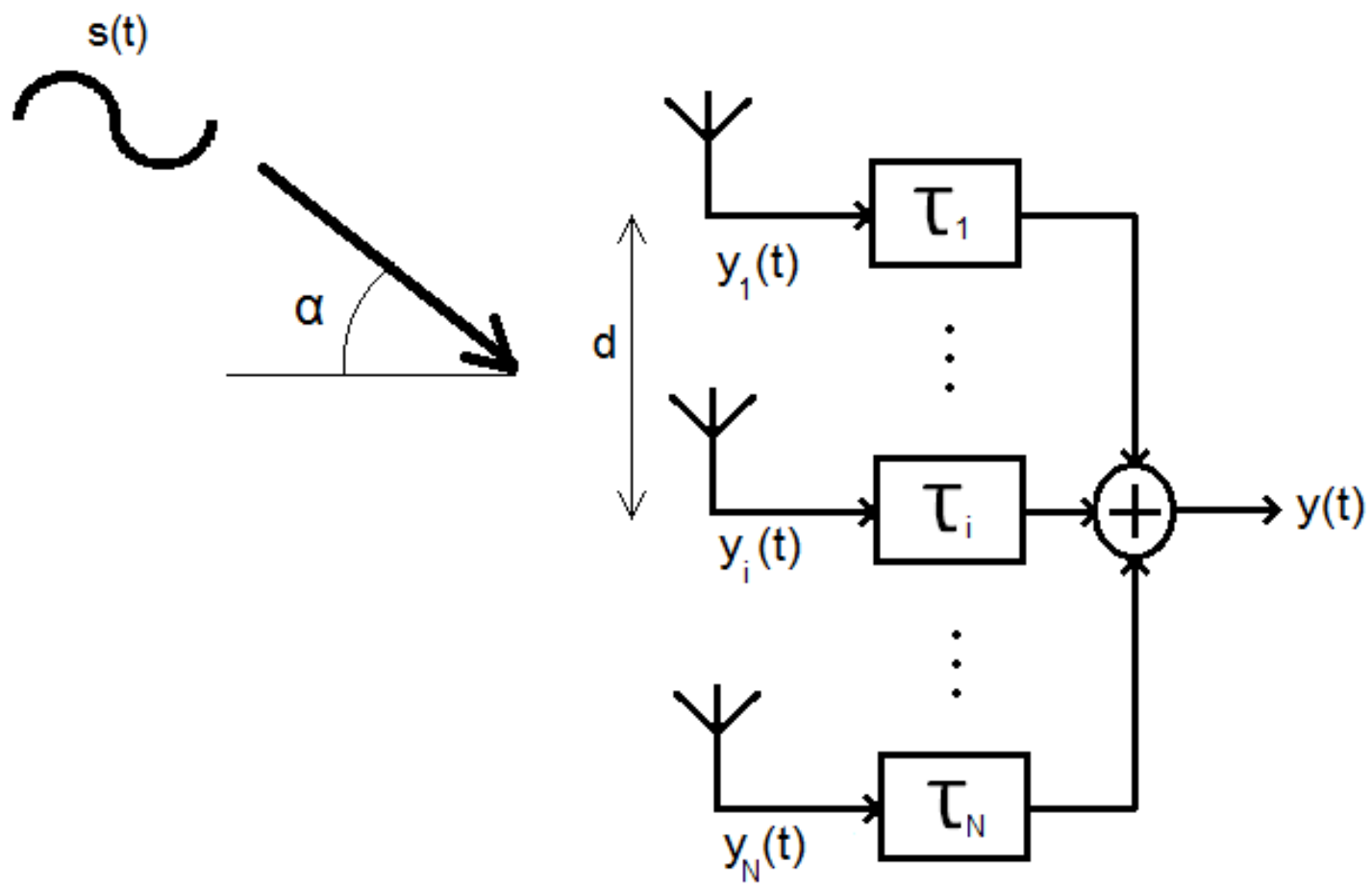

Fig. $5 \mathrm{~N}$-element array receiving an UWB symbol $\mathrm{s}(\mathrm{t})$

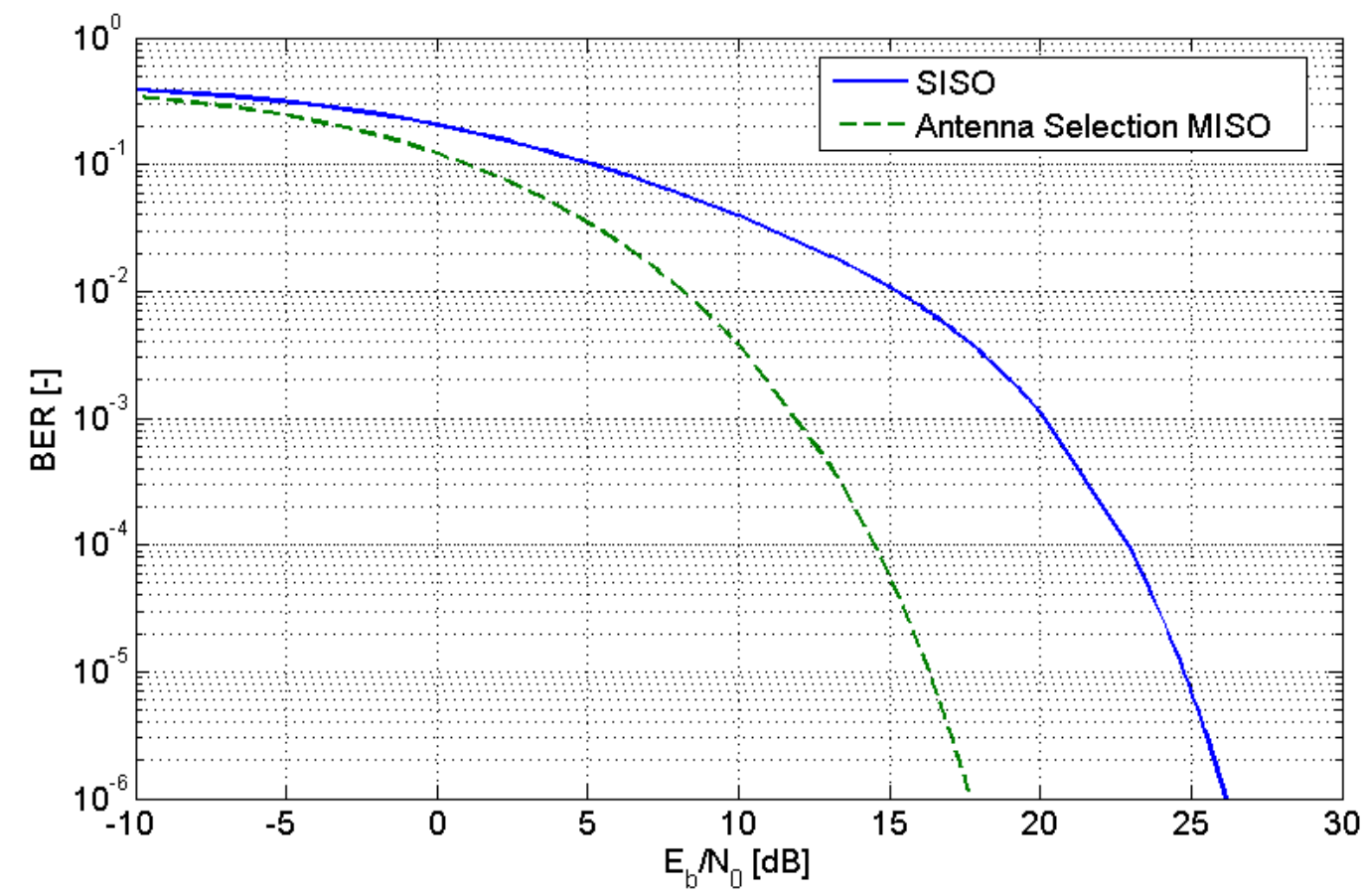

Fig. 6 Example comparison for gross BER performance (no channel coding) of WiMedia system for SISO system and MISO system with antenna selection for an empirical channel 


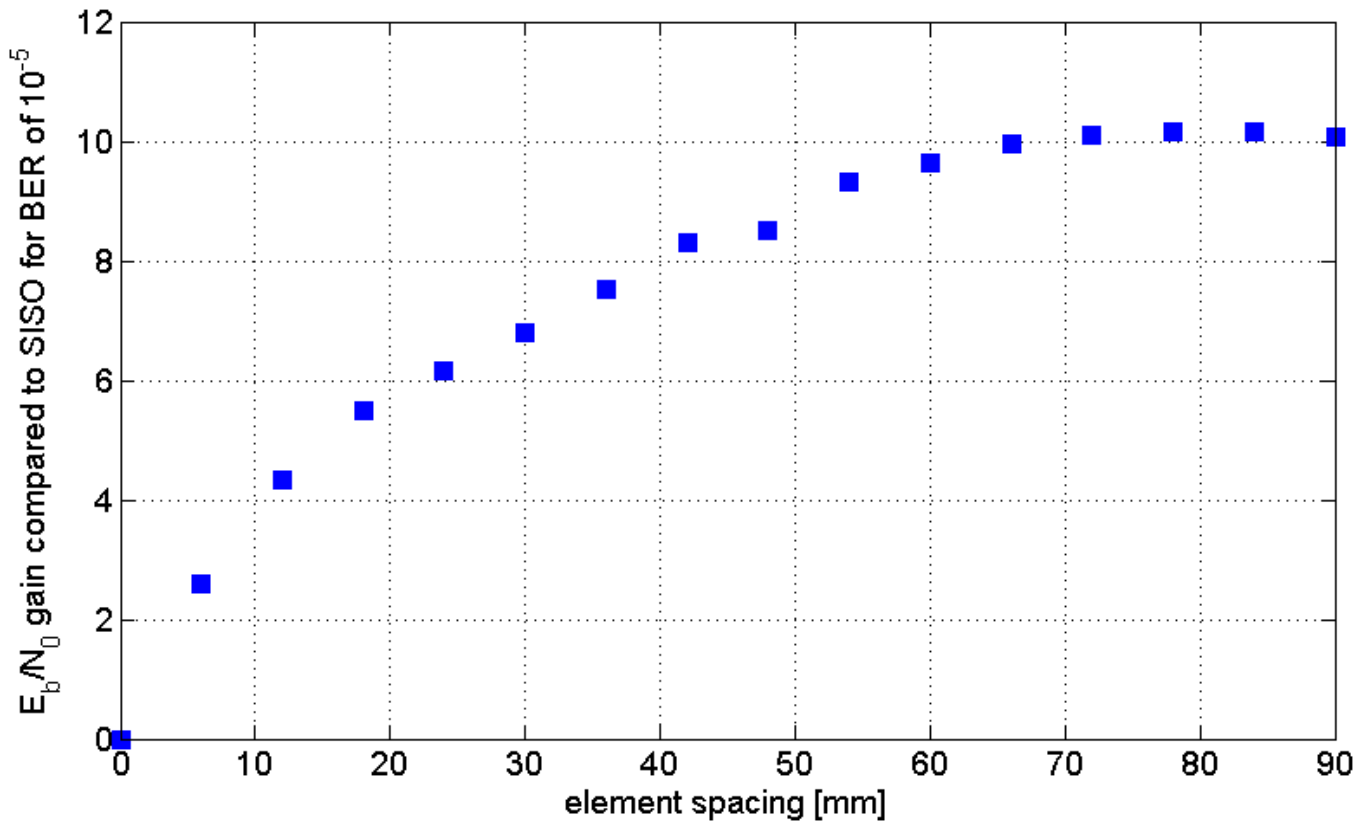

Fig. 7 Mean gain in gross BER performance (no channel coding) of WiMedia system MISO system with antenna selection compared to a SISO system as a function of element spacing

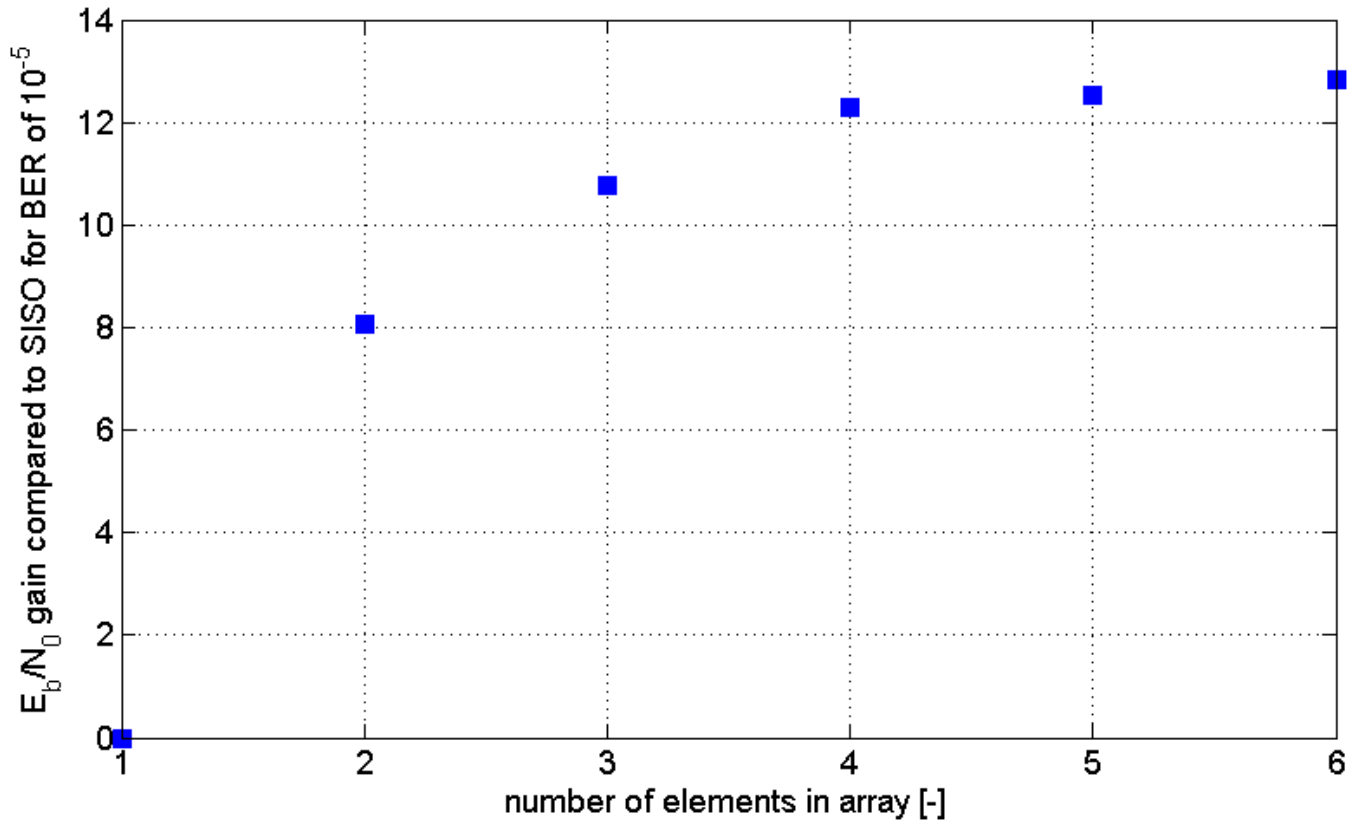

Fig. 8 Mean gain in gross BER performance (no channel coding) of WiMedia system MISO system with antenna selection compared to a SISO system as a function of number of elements 


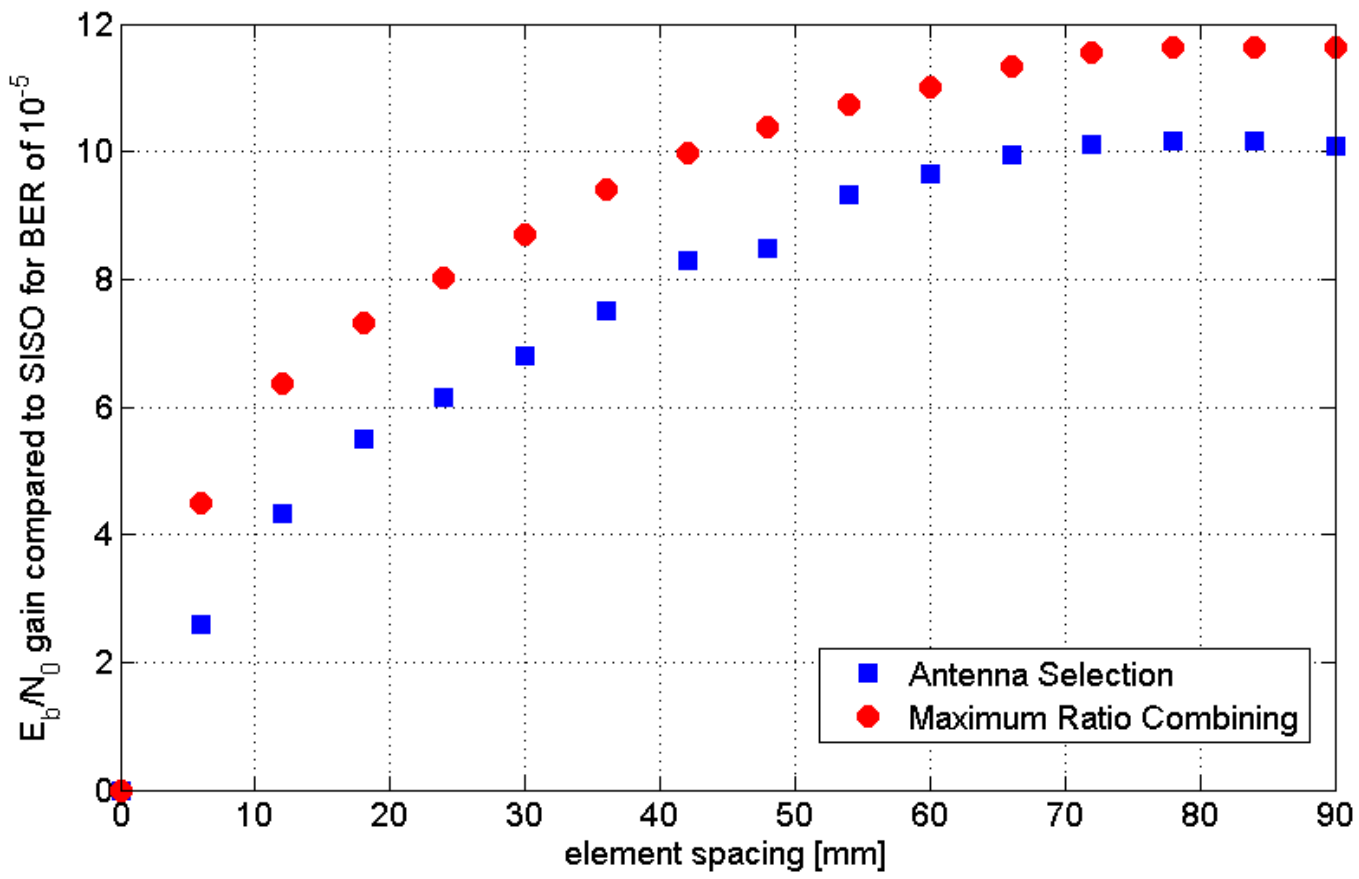

Fig. 9 Mean gain in gross BER performance (no channel coding) of WiMedia system SIMO system compared to SISO system as a function of element spacing comparing antenna selection and maximum ratio combining 